\title{
Slat Noise Prediction using Discontinuous Galerkin Method and Stochastic Turbulent Sound Source
}

\author{
M. Bauer* and R. Ewert ${ }^{\dagger}$ \\ DLR (German Aerospace Center), \\ Institute of Aerodynamics and Flow Technology, Technical Acoustics, \\ Lilienthalplatz 7, 38108 Braunschweig, Germany \\ email: marcus.bauer@dlr.de
}

\begin{abstract}
A promising way to predict airframe noise is the numerical solution of the Acoustic Perturbation Equations (APE) with the help of the Discontinuous Galerkin Method (DGM) and the Fast Random Particle Mesh (FRPM) method. The latter stochastically computes the turbulent source term of the APE, while the DGM reliably provides their spatial discretization, even on a flexible unstructured grid. The goal of the current work is to compute broadband slat noise of two different high-lift airfoil configurations in two dimensions with this approach. The dependency of the result on the resolution of both the FRPM- as well as the DG-grid is analysed, and the parameter sensitivity of the FRPM method is investigated. Furthermore, computed sound pressure spectra are compared to measured spectra, and computational times are examined.
\end{abstract}

\section{Introduction}

Airframe noise is generated through the transformation of turbulent energy into acoustic energy in the vicinity of edges, such as the trailing or side edge of an airfoil profile. It significantly contributes to the overall noise level of modern airliners, especially during the approach and landing phase of flight, when the engines are throttled back. Then, the main sources of airframe noise are the landing gear and the high-lift system with its deployed slats and flaps, which are all geometrically complex objects.

Broadband airframe noise as an important contribution to airframe noise can efficiently be computed with a hybrid CFD (Computational Fluid Dynamics)/CAA (Computational Aero Acoustics) approach, see figure 1. First, a CFD code computes the time-averaged flow around the object under consideration as a solution of the RANS (Reynolds Averaged Navier Stokes) equations. Second, a CAA code computes the unsteady generation and propagation of sound by solving the Acoustic Perturbation Equations (APE), ${ }^{14}$ a variant of the Linearized Euler Equations (LEE). Their unknowns depend both on space and time, whereas their coefficients are merely space-dependent, and are thus given by the preceeding RANS computation. The unsteady turbulent source term on the right-hand side of the APE is computed efficiently with the Fast Random Particle Mesh (FRPM) method, ${ }^{8}$ which stochastically synthesizes a turbulent velocity field from RANS data. Dealing with complex objects, unstructured computational grids are strongly preferred to block-structured ones. Considering the CAA side of the hybrid CFD/CAA approach, the Discontinuous Galerkin Method (DGM) may then be used to discretize the APE, since it is known to provide a robust, efficient, high-order accurate discretization even on unstructured grids.

Ref. 3 presented a 2D FRPM-DG method for CAA, where the employed DGM is based on nodal basis functions given in terms of Lagrange polynomials. ${ }^{7,22,23}$ First, computations with a model monopole sound source situated in a generic boundary layer proved this particular DGM to reliably predict sound refraction through a sheared mean flow field, which is an important aeroacoustic phenomenon. Solutions were stable and free from spurious numerical oscillations. Second, broadband slat noise of an airfoil with a deployed slat

\footnotetext{
${ }^{*}$ Research Engineer, Member AIAA

${ }^{\dagger}$ Research Engineer, Member AIAA, Team Lead Simulation Technologies
} 


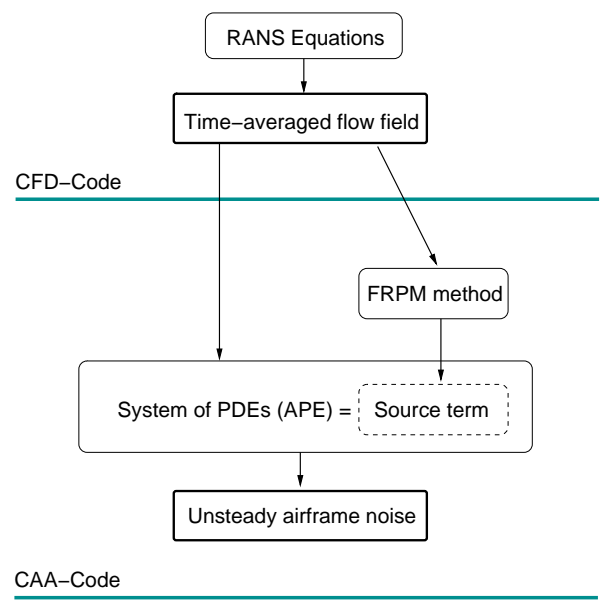

Figure 1. Hybrid CFD/CAA approach for efficient prediction of broadband airframe noise.

was computed in two dimensions. However, some closer investigation of the computed results, like e.g. the analysis of sound pressure spectra, is missing in Ref. 3.

The goal of the present work is to continue the research from Ref. 3. Slat noise of two different 2D high-lift configurations is computed with the FRPM-DG method. Basic properties of the method, like e.g. the grid dependency of the solution, are analysed, and computed sound pressure spectra are compared to measurements. In contrast to Ref. 3, a different source model is employed: The output of the FRPM method is directly interpreted as the fluctuating turbulent vorticity, and not as a streamfunction, from which the turbulent velocity and vorticity fluctuations follow through spatial differentiation. Like Ref. 3, the current work focuses on the CAA part of the hybrid CFD/CAA approach.

\section{Method}

\section{II.A. Acoustic Perturbation Equations (APE)}

Four different formulations of the Acoustic Perturbation Equations were proposed. ${ }^{14}$ The most popular APE-4 system, which is also considered here, reads in symbolic notation:

$$
\begin{aligned}
& \frac{\partial p^{\prime}}{\partial t}+c_{0}^{2} \nabla \cdot\left(\varrho_{0} \boldsymbol{u}^{\prime}+\boldsymbol{u}_{0} \frac{p^{\prime}}{c_{0}^{2}}\right)=c_{0}^{2} q_{c}, \\
& \frac{\partial \boldsymbol{u}^{\prime}}{\partial t}+\nabla\left(\boldsymbol{u}_{0} \cdot \boldsymbol{u}^{\prime}\right)+\nabla\left(\frac{p^{\prime}}{\varrho_{0}}\right)=\boldsymbol{q}_{m},
\end{aligned}
$$

where $t$ denotes time, $p$ pressure, $\varrho$ density, and $\boldsymbol{u}$ the velocity vector. A prime marks unknown time- and space-dependent perturbation variables, whereas index 0 indicates time-averaged, i.e. steady, mean flow quantities. The speed of sound is named $c_{0}$, and the right hand side symbols $q_{c}$ and $\boldsymbol{q}_{m}$ denote the sources.

The exact sources follow by rewriting the homogeneous Navier-Stokes Equations in primitive non-linear disturbance form, so that the LHS equals that from Eqs. (1). ${ }^{12,14}$ For vortex sound problems like airframe noise, all viscous, non-linear, and entropy-related terms of the source may be neglected, ${ }^{13,14}$ resulting in $q_{c}=0$ and $\boldsymbol{q}_{m}=-\boldsymbol{L}^{\prime}$, where $\boldsymbol{L}^{\prime}$ is the linear perturbed Lamb vector,

$$
\boldsymbol{L}^{\prime}=\boldsymbol{\omega}^{\prime} \times \boldsymbol{u}_{0}+\boldsymbol{\omega}_{0} \times \boldsymbol{u}^{\prime}
$$

$\boldsymbol{\omega}=\nabla \times \boldsymbol{u}$ denoting the vorticity vector. For a start, only the first part of the Lamb vector, $\boldsymbol{\omega}^{\prime} \times \boldsymbol{u}_{0}$, is retained. Accordingly, the output of the FRPM filter integral is directly interpreted as the perturbation vorticity. Using Cartesian coordinates $(x, y, z)$, the employed two-dimensional source $\boldsymbol{q}_{m}$ thus reads

$$
\boldsymbol{q}_{m}=-\left(\begin{array}{r}
-\omega_{3}^{\prime} v_{0} \\
\omega_{3}^{\prime} u_{0}
\end{array}\right),
$$


where the velocity vector is given by $\boldsymbol{u}=[u, v]^{\mathrm{T}}$, and $\omega_{3}$ denotes the $z$-component of the vorticity vector. Since the APE are linear, the principle of superposition holds, and the contribution of the second part of $\boldsymbol{L}^{\prime}$, $\boldsymbol{\omega}_{0} \times \boldsymbol{u}^{\prime}$, may easily be added later.

\section{II.B. DG Method}

The employed DGM is thoroughly described in Ref. 3. It provides the spatial discretization of the APE by way of nodal basis functions given in terms of Lagrange Polynomials of a certain degree $p .^{3,7,22,23}$ They both approximate the distribution of the perturbation quantities as well as that of the RANS mean flow variables. Consequently, products of those quantities are actually polynomials of degree $2 p$. However, truncation to a lower degree is advisable to save computational time. ${ }^{2,19}$ Making use of the Lagrangian nodes, products are efficiently truncated to degree $p$.

A polynomial degree of $p=3$ is used and time integration is performed by the standard explicit fourth order accurate Runge-Kutta scheme. Accordingly, the overall order of accuracy of the method is four, which was verified by convergence tests. The DG-APE method assumes constant sound speed $c_{0}$, since the ambient temperature is nearly constant in the considered cases of airframe noise. Furthermore, the method is currently limited to $2 \mathrm{D}$ and works on grids composed of triangular elements.

\section{II.C. FRPM Method}

II.C.1. Theory

The FRPM method ${ }^{8}$ synthesizes the perturbation vorticity $\omega_{3}^{\prime}$ of the two-dimensional source term, Eq. (3), from RANS data by spatially filtering a white noise field $\mathcal{U}$ like

$$
\omega_{3}^{\prime}(\boldsymbol{x}, t)=\iint_{A_{s}} \hat{A}\left(\boldsymbol{x}^{\prime}\right) G_{0}\left(\boldsymbol{x}, \boldsymbol{x}^{\prime}\right) \mathcal{U}\left(\boldsymbol{x}^{\prime}, t\right) d \boldsymbol{x}^{\prime}
$$

The integration area $A_{s}$ corresponds to the source patch in which the source term is computed, $\hat{A}$ is a local amplitude, and $G_{0}$ the filter kernel.

For incompressible flow, $\nabla \cdot \boldsymbol{u}_{0}=\mathbf{0}$, the white noise field $\mathcal{U}$ is defined by the properties ${ }^{8,9,11}$

$$
\begin{aligned}
\langle\mathcal{U}(\boldsymbol{x}, t)\rangle & =0, \\
\langle\mathcal{U}(\boldsymbol{x}, t) \mathcal{U}(\boldsymbol{x}+\boldsymbol{r}, t)\rangle & =\delta(\boldsymbol{r}), \\
\frac{D_{0}}{D t} \mathcal{U}(\boldsymbol{x}, t) & =0,
\end{aligned}
$$

where $\delta(\boldsymbol{r})$ is the Dirac delta function, and the substantial derivative is given by $\frac{D_{0}}{D t}=\frac{\partial}{\partial t}+\boldsymbol{u}_{0} \cdot \nabla$ in terms of the mean velocity field $\boldsymbol{u}_{0}$ from RANS. The brackets $\langle\ldots\rangle$ denote an ensemble average, which actually corresponds to the time average, since ergodic problems are considered. The time average is in turn denoted by an overbar in this article.

The filter kernel $G_{0}$ corresponds - roughly speaking - to a Gaussian kernel $G_{1}$ characterized by the filter width $l_{s}$,

$$
G_{1}\left(\boldsymbol{x}, \boldsymbol{x}^{\prime}\right)=\exp \left(-\frac{\pi}{2} \frac{\left(\boldsymbol{x}-\boldsymbol{x}^{\prime}\right)^{2}}{l_{s}^{2}}\right),
$$

see section II.C.2 for details. $G_{1}$ can advantageously be split into a product of two one-dimensional kernels like

$$
G_{1}\left(\boldsymbol{x}, \boldsymbol{x}^{\prime}\right)=\underbrace{\exp \left(-\frac{\pi}{2} \frac{\left(x-x^{\prime}\right)^{2}}{l_{s}^{2}}\right)}_{G_{1}^{x}\left(x, x^{\prime}\right)} \cdot \underbrace{\exp \left(-\frac{\pi}{2} \frac{\left(y-y^{\prime}\right)^{2}}{l_{s}^{2}}\right)}_{G_{1}^{y}\left(y, y^{\prime}\right)} .
$$

A spatially constant filter width $l_{s}$ is employed in this work.

The filter amplitude obeys

$$
\hat{A}=\sqrt{k} / l_{s}^{2},
$$

where $k$ denotes the turbulent kinetic energy from RANS. Eq. (10) is valid if the filter width $l_{s}$ is constant as stated above, and its derivation is outlined in the first part of the Appendix. Next to the usually 
employed local dependency $\hat{A}=\hat{A}\left(\boldsymbol{x}^{\prime}\right)=\sqrt{k\left(\boldsymbol{x}^{\prime}\right)} / l_{s}^{2}$ the alternative formulation $\hat{A}=\hat{A}(\boldsymbol{x})=\sqrt{k(\boldsymbol{x})} / l_{s}^{2}$ is considered, too. This alternative formulation exactly realizes a desired local source variance. Using the standard formulation, the realized source variance is the target source variance implicitly smoothed out with a standardized Gaussian filter kernel of width $l_{s}$. This allows to attenuate the impact of potential inconsistencies in RANS data. Both formulations were tested for trailing edge noise in Ref. 11.

\section{II.C.2. Discretization}

The source is computed at the grid points $\boldsymbol{x}_{i j}$ of a Cartesian background mesh, which spans the so-called (source) patch, see figure 2. The numerical quadrature to realize the filter integral Eq. (4) is given by

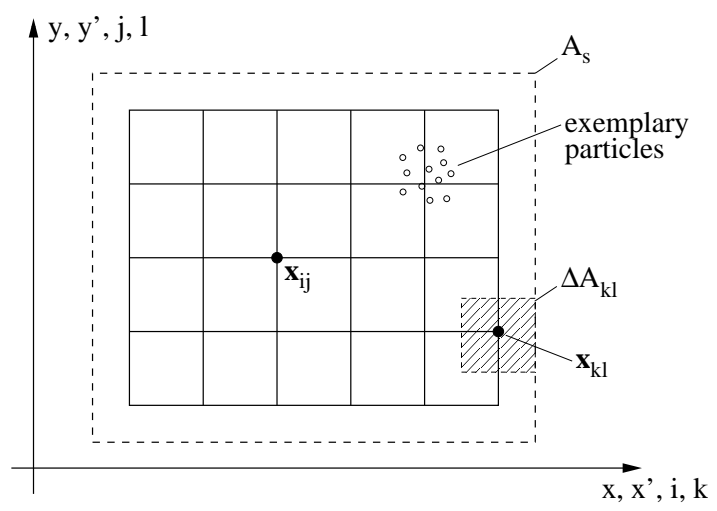

Figure 2. Sketch of FRPM source patch.

$$
\omega_{3}^{\prime}\left(\boldsymbol{x}_{i j}, t\right)=\sum_{m=1}^{N_{P}} \hat{A}\left(\boldsymbol{x}_{m}\right) G_{0}\left(\boldsymbol{x}_{i j}, \boldsymbol{x}_{m}\right) r_{m}(t)
$$

with

$$
r_{m}(t):=\iint_{\Delta A_{m}} \mathcal{U}\left(\boldsymbol{x}^{\prime}\right) \mathrm{d} \boldsymbol{x}^{\prime} .
$$

The random field $\mathcal{U}$ is approximated by a cloud of $N_{P}$ particles situated at arbitrary locations $\boldsymbol{x}_{m}$ on the patch. They are evenly distributed at the beginning of a computation. Reasonably, no particles are put into objects, though. They drift with the local mean flow velocity. Therefore, the values of $\boldsymbol{u}_{0}$ are bilinearly interpolated from the cell corners to the actual position of a particle inside its hosting cell at each time step. The convection is then computed with the explicit, second order accurate Adams-Bashforth multistep method. Particles that leave the patch are replaced by new ones seeded at inflow boundaries.

A control area $\Delta A_{m}$ surrounds each particle. Assuming non-overlapping control areas, evenly distributed particles, and incompressible mean flow they are all of the same constant size $\Delta A_{m}=A_{s} / N_{P}$.

A random number $r_{m}$ is associated to each of the particles, representing the white-noise field integrated over the area $\Delta A_{m}$. The properties of the random numbers can be deduced from Eqs. $(5,6)$ in conjunction with Eq. (12). From Eq. (5) follows that

$$
\left\langle r_{m}(t)\right\rangle=0
$$

and from Eq. (6) that

$$
\left\langle r_{m}(t) r_{n}(t)\right\rangle=\Delta A_{m} \delta_{n m}
$$

i.e., their mean value vanishes, and their variance is proportional to $\Delta A_{m}$, which is constant in incompressible mean flow as stated above.

The spatial filter $G_{0}$ can be split into the convolution of two filter kernels of length scale $l_{1}$ and $l_{2}$,

$$
G_{0}\left(\boldsymbol{x}_{i j}, \boldsymbol{x}_{m}, l_{0}\right)=\int G_{1}\left(\boldsymbol{x}_{i j}-\boldsymbol{x}^{\prime}, l_{1}\right) G_{2}\left(\boldsymbol{x}^{\prime}, \boldsymbol{x}_{m}, l_{2}\right) \mathrm{d} \boldsymbol{x}^{\prime}=\left(G_{1} * G_{2}\right)\left(\boldsymbol{x}_{i j}, \boldsymbol{x}_{m}\right) .
$$

If a Gaussian kernel $G_{0}$ is used, the resulting filtered quantity realizes a correlation $R_{0}=G_{0} * G_{0}$. From a Gaussian filter a Gaussian correlation function results. Based on a filter kernel which is generated by the 
convolution of two filters $G_{1}$ and $G_{2}$ the resulting correlation becomes $R=R_{1} * R_{2}$, where $R_{1}=G_{1} * G_{1}$ and $R_{2}=G_{2} * G_{2}$. If $R_{1}$ and $R_{2}$ represent Gaussian correlations of length scale $l_{1}$ and $l_{2}$, respectively, the resulting correlation $R_{0}$ is a Gaussian as well, and its length scale becomes $l_{0}=\sqrt{l_{1}^{2}+l_{2}^{2}}$. Furthermore, the property of a multi-dimensional Gaussian filter to separate into a sequence of 1D filtering operations, also holds for the right-hand side convolutions in Eq. (15). It was discussed in Ref. 8, that a filter of the form

$$
g(x)=\left\{\begin{array}{lll}
1-\frac{|x|}{\Delta} & \text { for } & |x| \leq \Delta \\
0 & \text { for } & |x|>\Delta
\end{array}\right.
$$

yields a cubic spline correlation function $r=g * g$ that closely resembles a Gaussian of length scale $3 \Delta / 4$, i.e. (with $\xi:=x / \Delta)$

$$
r(\xi)=\left\{\begin{array}{ll}
1-\frac{3}{2} \xi^{2}+\frac{3}{4} \xi^{3} & \text { for } \quad|\xi|<1 \\
\frac{1}{4}(2-\xi)^{3} & \text { for } 1 \leq|\xi|<2 \\
0 & \text { for } 2 \leq|\xi|
\end{array} .\right.
$$

In the FRPM approach, a multi-dimensional filter $G_{2}\left(\boldsymbol{x}, \boldsymbol{x}^{\prime}\right)=g\left(x-x^{\prime}\right) g\left(y-y^{\prime}\right)$ is applied together with a Gaussian filter $G_{1}$ on the auxiliary mesh. The convolution integral Eq. (15) is approximated by the discrete convolution

$$
G_{0}\left(\boldsymbol{x}_{i j}, \boldsymbol{x}_{m}\right)=\sum_{k} \sum_{l} G_{1}\left(\boldsymbol{x}_{i j}-\boldsymbol{x}_{k l}\right) G_{2}\left(\boldsymbol{x}_{k l}, \boldsymbol{x}_{m}\right) \Delta A_{k l}
$$

where $\Delta A_{k l}$ represents a control area of the auxiliary mesh, recall figure 2 .

By combining Eq. (11) and Eq. (18) and changing the order of the summations, the procedure becomes

$$
\omega_{3}^{\prime}\left(\boldsymbol{x}_{i j}, t\right)=\sum_{k} \sum_{l} G_{1}\left(\boldsymbol{x}_{i j}-\boldsymbol{x}_{k l}\right) \Delta A_{k l} \sum_{m=1}^{N_{P}} G_{2}\left(\boldsymbol{x}_{k l}, \boldsymbol{x}_{m}\right) \hat{A}\left(\boldsymbol{x}_{m}\right) r_{m}(t) .
$$

Practically, the rightmost summation over all $N_{P}$ particles with filter $G_{2}\left(\boldsymbol{x}, \boldsymbol{x}^{\prime}\right)=g\left(x-x^{\prime}\right) g\left(y-y^{\prime}\right)$ means to run over all cells of the auxiliary mesh and to bilinearly interpolate the random values of all particles contained in the current hosting cell onto its four cell vertices. The final summations over $k$ and $l$ are carried out on the auxiliary mesh. That is, the first filter $G_{2}$ interpolates the random values of the particles onto the vertices of the auxiliary mesh. The second filter $G_{1}$ with length scale $l_{s}$ accomplishes a filtered field on the auxiliary mesh. The first filter step is realized with a filter length scale $\Delta$, which corresponds to the mesh spacing of the auxiliary mesh. Altogether a correlation length $l=\sqrt{l_{s}^{2}+(3 \Delta / 4)^{2}}$ is achieved. When $\Delta$ is sufficiently smaller than $l_{s}$, it has almost no impact on the realized length scale, i.e. $l \approx l_{s}$. For smaller $l_{s}$, e.g. if it is given by the variable RANS length scale, the resulting length scale is automatically limited to $3 \Delta / 4$.

The filter amplitude $\hat{A}$ is stored in the grid points $\boldsymbol{x}_{k l}$ of the background mesh. According to Eq. (19), however, it has to be available at the particle locations $\boldsymbol{x}_{m}$, i.e., it actually has to be interpolated from the background mesh onto the particles at every time step. In this work, $\hat{A}$ is assumed to change only weakly in a cell, and $\hat{A}\left(\boldsymbol{x}_{m}\right)$ is approximated by $\hat{A}\left(\boldsymbol{x}_{k l}\right)$ to circumvent this additional interpolation. Thus, Eq. (19) simplifies to

$$
\omega_{3}^{\prime}\left(\boldsymbol{x}_{i j}, t\right)=\sum_{k} \sum_{l} \hat{A}\left(\boldsymbol{x}_{k l}\right) G_{1}\left(\boldsymbol{x}_{i j}-\boldsymbol{x}_{k l}\right) \underbrace{\Delta A_{k l} \sum_{m=1}^{N_{P}} G_{2}\left(\boldsymbol{x}_{k l}, \boldsymbol{x}_{m}\right) r_{m}(t)}_{\mathcal{W}\left(\boldsymbol{x}_{k l}, t\right)} .
$$

In the case of the alternative local dependency $\hat{A}=\hat{A}(\boldsymbol{x})$, Eq. (20) in turn becomes

$$
\omega_{3}^{\prime}\left(\boldsymbol{x}_{i j}, t\right)=\hat{A}\left(\boldsymbol{x}_{i j}\right) \sum_{k} \sum_{l} G_{1}\left(\boldsymbol{x}_{i j}-\boldsymbol{x}_{k l}\right) \mathcal{W}\left(\boldsymbol{x}_{k l}, t\right)
$$

No matter which formulation is chosen for $\hat{A}$, the filtering with kernel $G_{1}$ on the auxiliary mesh is fast and efficient as explained in the second part of the Appendix. 


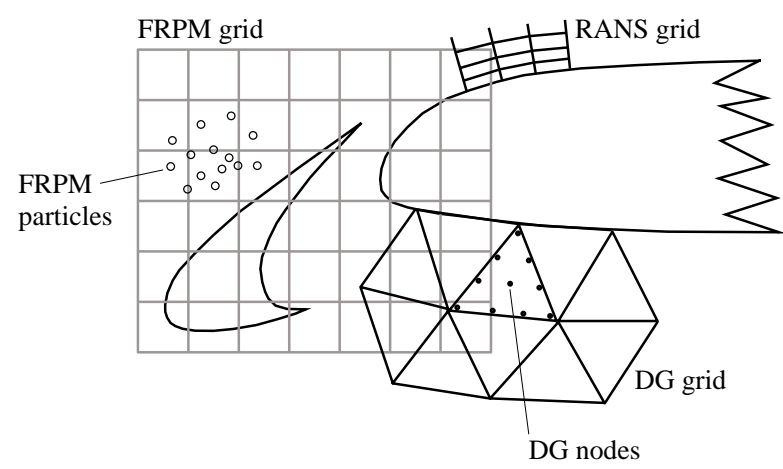

Figure 3. Sketch of different entities of hybrid CFD/CAA approach in source region around a deployed slat.

\section{II.D. Practical Realization}

Figure 3 illustrates the various entities of the hybrid CFD/CAA approach in a source region, and table 1 explains the spatial interpolations between them. In fact, the interpolations are all linear or bilinear and consequently second order accurate. The inverse distance algorithm mentioned in table 1 may improve the interpolation of mean density and mean pressure from a RANS grid onto a DG grid along curved walls using TecPlot. Apart from the perturbation vorticity $\omega_{3}^{\prime}$, the whole source vector $\boldsymbol{S}$ is actually computed on the

Table 1. Interpolations employed for hybrid CFD/CAA approach.

\begin{tabular}{c|ll|l|l} 
interpolated variables & from ... & onto ... & how ? & when ? \\
\hline $\begin{array}{c}\text { mean flow quantities } \\
\text { mean flow quantities }\end{array}$ & RANS grid & FRPM grid & TecPlot (linear) & preprocessing \\
mean velocity & FRPM grid & DG grid (all nodes) & $\begin{array}{l}\text { TecPlot (linear and/or } \\
\text { inverse distance) } \\
\text { preprocessing }\end{array}$ & each time step \\
random values & particles & FRPM grid & bilinearly & each time step \\
source vector & FRPM grid & $\begin{array}{l}\text { DG grid (only nodes } \\
\text { in source patch) }\end{array}$ & bilinearly & each time step
\end{tabular}

FRPM grid at each time step. It is then bilinearly interpolated onto those DG nodes which are located in the patch domain.

Both the FRPM method as well as the DG method were implemented in the programming language Fortran 90/95. A clear and well-defined interface was established between the two codes. Principally, not more than three statements were added to the DG control routine to realize the coupling with the FRPM code, namely:

1. USE frpm_mod

2. CALL Init_frpm

3. CALL Calc_frpm.

Module frpm_mod contains the two procedures Init_frpm and Calc_frpm. Init_frpm is called by the DG code once before the time loop to initialize FRPM and to prepare the bilinear interpolation of the source term onto the DG nodes in the patch area. Calc_frpm is repeatedly called from within the DG time loop to compute the new source term on the background mesh and to interpolate it onto the respective DG nodes. The actual FRPM procedures are in turn called from Init_frpm and Calc_frpm.

The maximum time step size $d t$ from $\mathrm{DG}^{3}$ is usually smaller than that from FRPM and was used for FRPM, too. The FRPM time step size is only limited by the demand that no particle be convected farther than one FRPM cell during a time step. 


\section{II.E. Nondimensionalization, Calculation of Spectra}

Dimensionless quantities were used in all FRPM-DG simulations and most of the coordinates, dimensions and variables in this paper are dimensionless, too, except for acoustic spectra, which are based on SI-units. Dimensionless quantities are related to dimensional quantities marked by superscript ${ }^{*}$ or subscript $\infty$ like

$$
t=t^{*} \frac{c_{\infty}}{L^{*}}, \quad \boldsymbol{x}=\frac{\boldsymbol{x}^{*}}{L^{*}}, \quad \varrho=\frac{\varrho^{*}}{\varrho_{\infty}}, \quad \boldsymbol{u}=\frac{\boldsymbol{u}^{*}}{c_{\infty}}, \quad p=\frac{p^{*}}{\varrho_{\infty} c_{\infty}^{2}}, \quad k=\frac{k^{*}}{c_{\infty}^{2}},
$$

where the coordinate vector is given by $\boldsymbol{x}=[x, y]^{\mathrm{T}}$. The dimensional reference quantities are a certain length $L^{*}$ as well as the density $\varrho_{\infty}$ and the speed of sound $c_{\infty}$ at infinity.

The spectra show levels calculated from the PSD (Power Spectral density) $\Phi(f)$ of the dimensional pressure perturbations. $\Phi(f)$ obeys

$$
\int_{0}^{\infty} \Phi(f) d f=\overline{p^{*}(t)^{2}}
$$

The ordinates of the spectra show $20 \log \left(\sqrt{\Phi(f) f_{\text {ref }}} / p_{\text {ref }}\right) \mathrm{dB}$, where $f_{\text {ref }}=1 \mathrm{~Hz}$ and $p_{\text {ref }}=2.0 \cdot 10^{-5} \mathrm{~Pa}$.

\section{Results}

\section{III.A. FREQUENZ High-Lift Airfoil Configuration}

\section{III.A.1. Foreword}

Slat noise of the high-lift configuration from the German national project FREQUENZ was computed. The geometry is a two-element profile consisting of an airfoil with a deployed slat, but for simplicity without a deployed flap. This rather simple geometry is well-suited to investigate basic properties of the method, and had already been considered in Ref. 3 .

\section{III.A.2. Setup}

Figure 4 shows the two-element profile and the employed coordinate systems. The APE were solved by the DG code in terms of the $(x, y)$ coordinates, whereas the polar coordinates $(r, \theta)$ were used for data postprocessing. The coordinate origin is at the leading edge of the clean airfoil or approximately at the deployed slat's trailing edge. The airfoil configuration was situated in cold flow of free stream Mach number

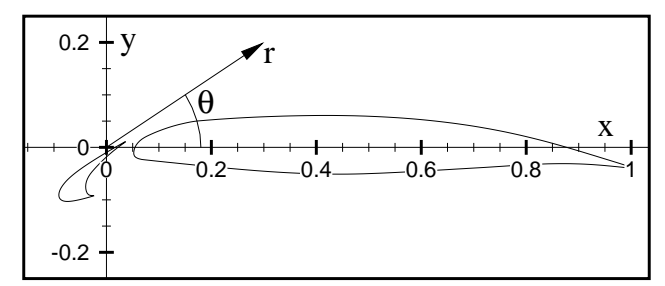

Figure 4. Coordinates at FREQUENZ geometry.

$M a=0.166$ at an angle of attack of $\alpha=13^{\circ}$. The reference quantities used for non-dimensionalization according to Eqs. (22) were the stowed chord of $L^{*}=0.4 \mathrm{~m}$, as well as the density $\varrho_{\infty}=1.21 \frac{\mathrm{kg}}{\mathrm{m}^{3}}$ and the speed of sound $c_{\infty}=342 \frac{\mathrm{m}}{\mathrm{s}}$ at infinity. Accordingly, the Reynolds number based on $L^{*}$ was $R e \approx 1.5 \cdot 10^{6}$.

The two-dimensional RANS solution was calculated by C.K.Appel (department AS-TA) with DLR's wellestablished Finite Volume CFD code TAU using a Reynolds Stress turbulence model. The hybrid RANS grid consisted of 80.325 quadrangles to properly resolve the boundary layers, and 101.243 triangles to fill the rest of the computational domain, which was circular and had a farfield extension of $r \approx 47$.

Four different FRPM grids were tested, see table 2. Each had a different resolution, but their extension was always $-0.1 \leq x \leq 0.2,-0.12 \leq y \leq 0.08$. Furthermore, the recommended number of five particles per cell $^{8}$ was engaged in each case, so that the particle count was e.g. 300.000 on the medium-fine grid consisting 
of 60.000 cells. To avoid spurious noise, ${ }^{4,13}$ the source term was smoothly faded out at the downstream patch boundary starting from $x=0.1$. The spatially constant filter length scale was usually set to $l_{s}=0.003$.

Table 2. Parameters of FRPM grids for FREQUENZ geometry; $I$ and $J$ denote the number of cells in $x$ - and $y$-direction, respectively, and $\Delta=\Delta x=\Delta y$ holds for the cell size.

\begin{tabular}{l|cccc} 
& $I$ & $J$ & $I \cdot J$ & $\Delta$ \\
\hline very coarse grid & 75 & 50 & 3.750 & 0.004 \\
coarse grid & 150 & 100 & 15.000 & 0.002 \\
medium-fine grid & 300 & 200 & 60.000 & 0.001 \\
fine grid & 600 & 400 & 240.000 & 0.0005
\end{tabular}

Four different DG grids were employed to study the dependence of the acoustic result on the DG grid resolution. The computational DG domain always had a diameter of four, and sharpened slat trailing edges were employed, see section III.A.7. All grids were refined in the boundary layer along the main element and in the source region, i.e., around the slat. Table 3 lists important grid parameters. For a better understanding: The dimensionless wavelength of a sound wave of, say, $f=10 \mathrm{kHz}$ would be $\lambda \approx 0.09$ in quiescent air here.

Table 3. Parameters of DG grids used for convergence studies at FREQUENZ geometry; $E$ denotes overall number of elements, $L_{\mathrm{src}}$ is the approximate length of the triangle edges in the source region, $L_{\text {main }}$ in the main element boundary layer, and $L_{\text {rest }}$ in the rest of the computational domain.

\begin{tabular}{l|cccc} 
& $E$ & $L_{\text {src }}$ & $L_{\text {main }}$ & $L_{\text {rest }}$ \\
\hline very coarse grid & 15.865 & 0.00450 & 0.01000 & 0.0450 \\
coarse grid & 20.945 & 0.00450 & 0.00450 & 0.0450 \\
medium-fine grid & 42.561 & 0.00300 & 0.00300 & 0.0300 \\
fine grid & 71.238 & 0.00225 & 0.00225 & 0.0225
\end{tabular}

Figure 5 depicts the very coarse grid. It is a variant of the coarse grid with an approximately two times coarser resolution in the main element's boundary layer. Furthermore, next to the slat trailing edges, also the main element trailing edge, which actually has a thickness of $d \approx 0.003$, was sharpened in this very grid. As is well-known, one important advantage of unstructured grids over block-structured ones is the
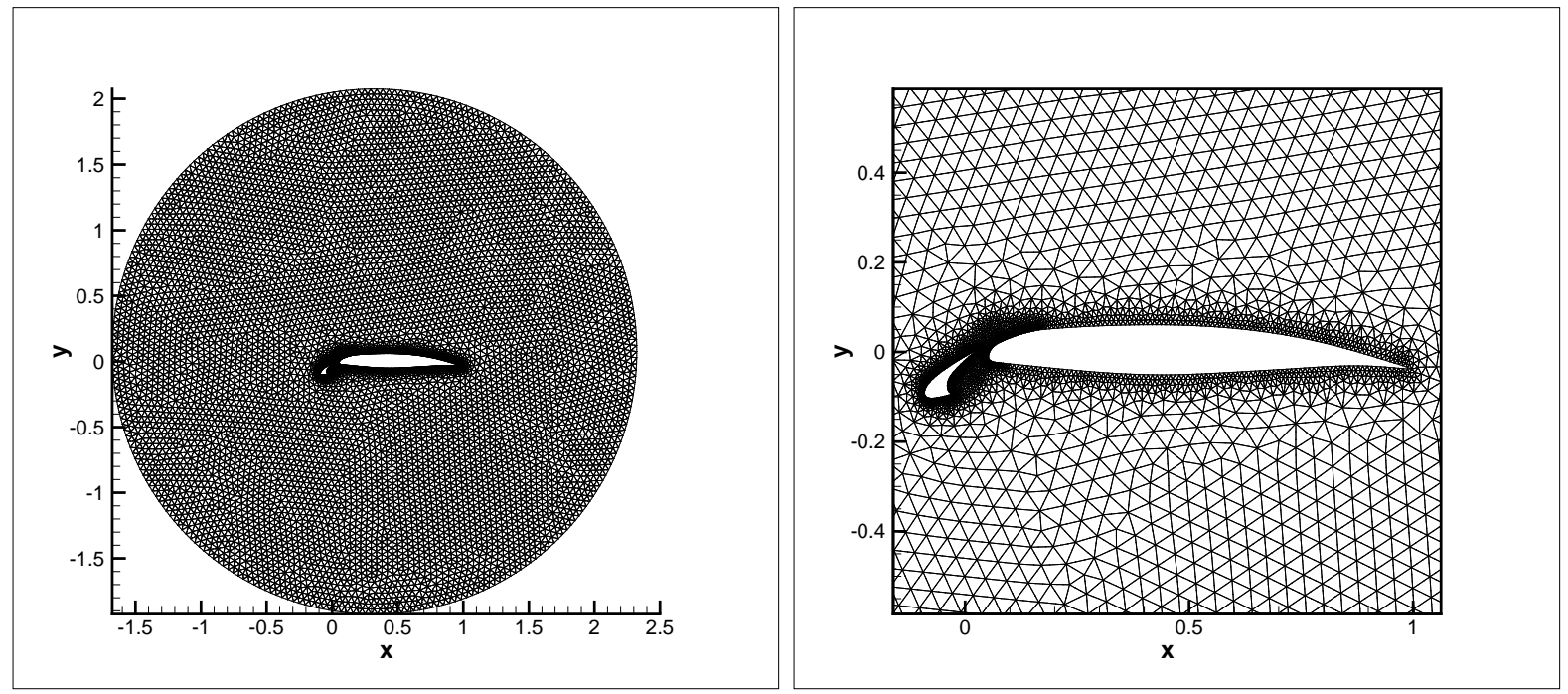

Figure 5. Coarsest DG grid for FREQUENZ geometry consisting of $E=15.865$ elements; left: overall grid; right: close-up; next to the slat edges, also the main element trailing edge was sharpened in this very grid.

much easier, much faster, and automated manner to generate grids around complex geometries. Another 
significant advantage becomes evident from figure 5: local grid refinement, like e.g. in the slat region, can be performed very conveniently, and indeed remains local and does not extend into the farfield.

\section{III.A.3. RANS Impressions}

Figure 6 depicts the RANS solutions for the Mach number $M a$ and the turbulent kinetic energy $k$ in the slat region. The turbulent kinetic energy was calculated as half of the trace of the Reynolds stress tensor. The pictures look similar to respective data extracted from a LES (Large Eddy Simulation) carried out for the same geometry. ${ }^{18}$
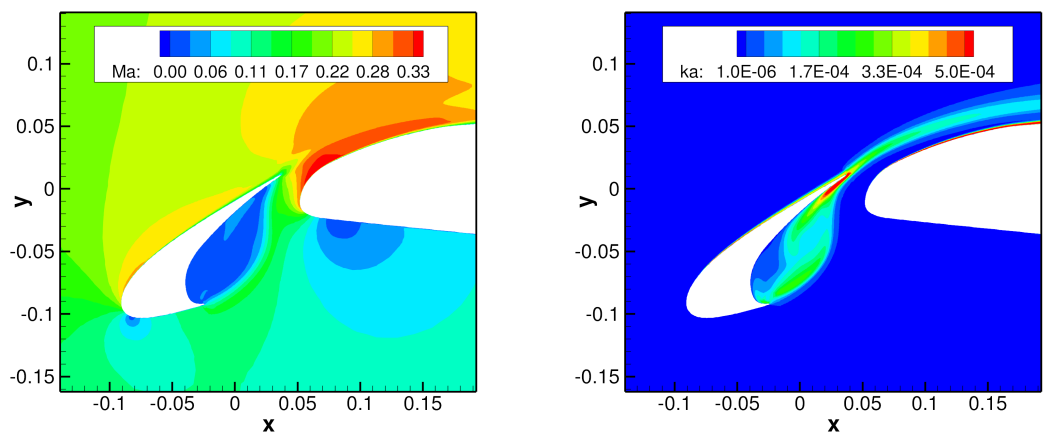

Figure 6. Distributions of Mach number (left) and of turbulent kinetic energy (right) around slat of FREQUENZ geometry on RANS grid.

\section{III.A.4. FRPM Impressions}

Figure 7 displays the distribution of the filter amplitude $\hat{A}$ as well as an instantaneous distribution of the generated vorticity fluctuations $\omega_{3}^{\prime}$ on the medium-fine FRPM grid consisting of 60.000 cells. To generate $\omega_{3}^{\prime}$, a local dependency $\hat{A}=\hat{A}\left(\boldsymbol{x}^{\prime}\right)$ and a filter width $l_{s}=0.003$ were employed. The strip of $k$ or $\hat{A}$ associated to the thin boundary layer along the main element suction side was blanked out, as its contribution to the generated airframe noise was found to be negligible. It may be checked in the future, how well the mean square of $\omega_{3}^{\prime}$ meets its target value of $\overline{\omega_{3}^{\prime 2}}=\hat{A}^{2} l_{s}^{2}$, Eq. (38).
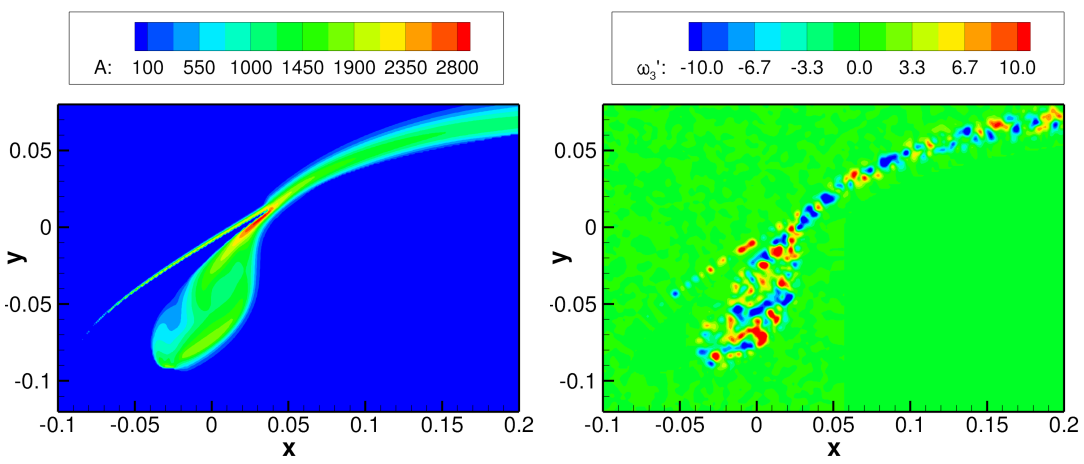

Figure 7. Distributions of filter amplitude (left) and of instantaneous vorticity fluctuations (right) on medium-fine FRPM grid for FREQUENZ geometry.

\section{III.A.5. Pressure Perturbation Field}

Figure 8 shows an instantanuous pressure perturbation field computed on the very coarse DG grid from table 3 using the medium-fine FRPM grid. Solutions from other grids look very similar. Hardly any sound waves are visible under an angle of $\theta=210^{\circ}$ ahead of the slat, and the sound waves propagating in upstream direction are compressed by the mean flow field. 


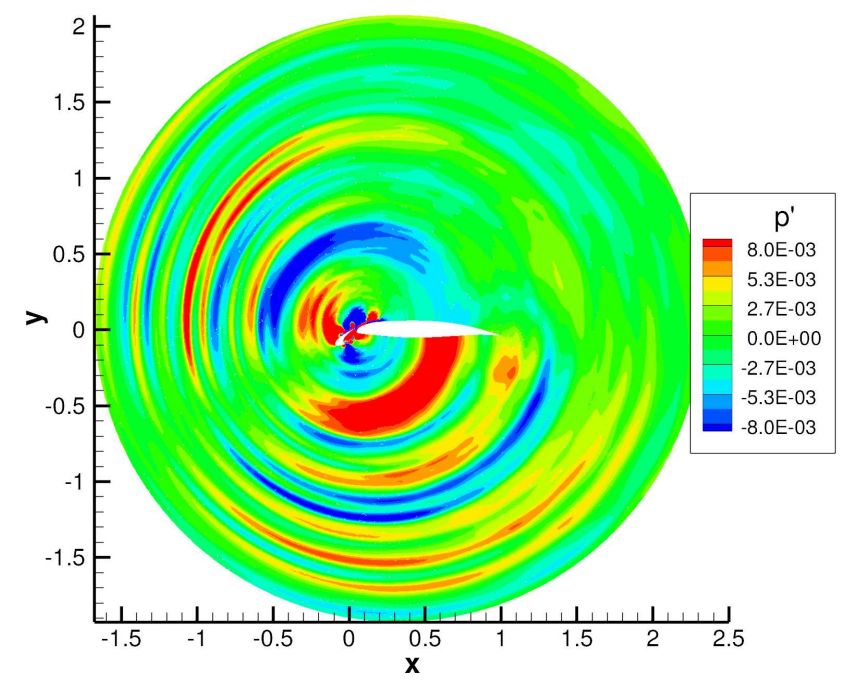

Figure 8. Snapshot of pressure perturbation field computed for FREQUENZ airfoil configuration on very coarse DG grid.

\section{III.A.6. DG Grid Resolution Studies}

Figure 9 compares sound pressure spectra obtained on the four DG grids from table 3 using the medium-fine FRPM grid. There are harly any differences below $10 \mathrm{kHz}$ and, naturally, higher cut-off frequencies are obtained with the finer grids.
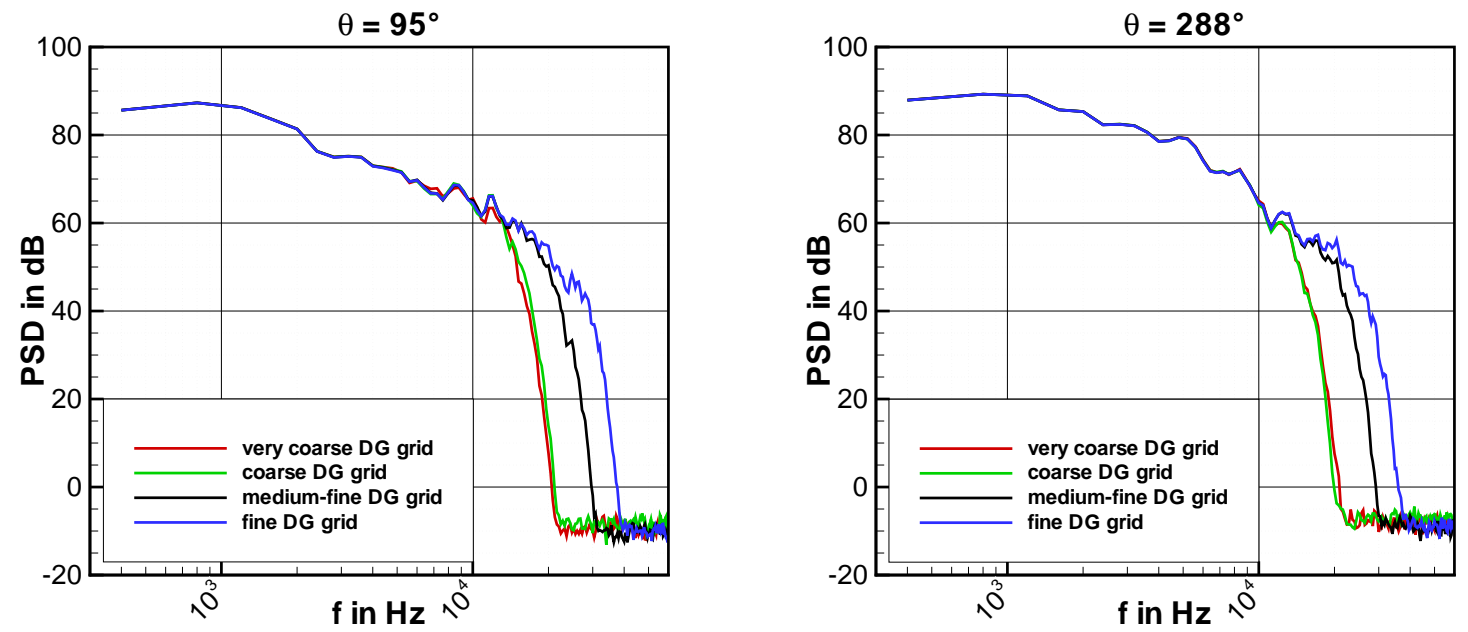

Figure 9. Sound pressure spectra computed for FREQUENZ profile on different DG grids; left: receiving point at $(r, \theta)=\left(1.5,95^{\circ}\right)$; right: receiving point at $(r, \theta)=\left(1.5,288^{\circ}\right)$; please refer to section III.A.10 for an explanation of the absolute levels.

This is also well illustrated by sound pressure level directivities as shown in figure 10. The underlying pressure signal was recorded at 360 virtual microphones along path $r=1.5$, so that $\Delta \theta=1^{\circ}$. The overall sound pressure level is practically identical for all angles on all grids, i.e., even the very coarse grid predicts it accurately. As mentioned above, this grid employs a particularly coarse resolution of the boundary layer along the main element plus a sharpened main element's trailing edge. Considering the levels at distinct frequencies, there are only little differences between the various grids up to $10 \mathrm{kHz}$. The solution from 
a)
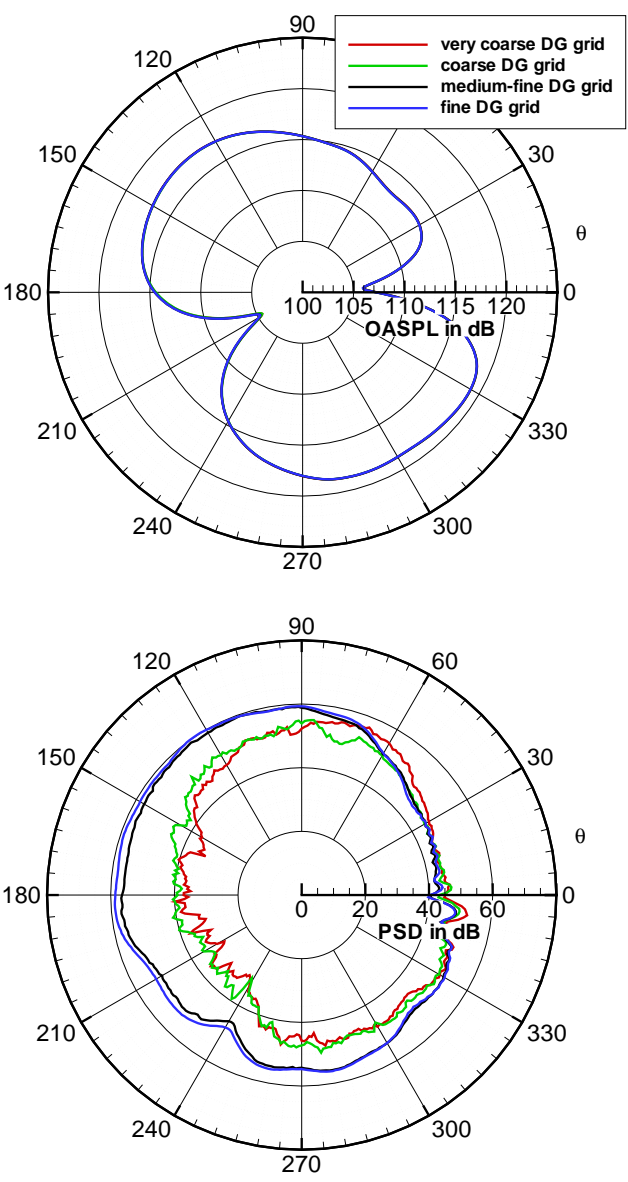

b)
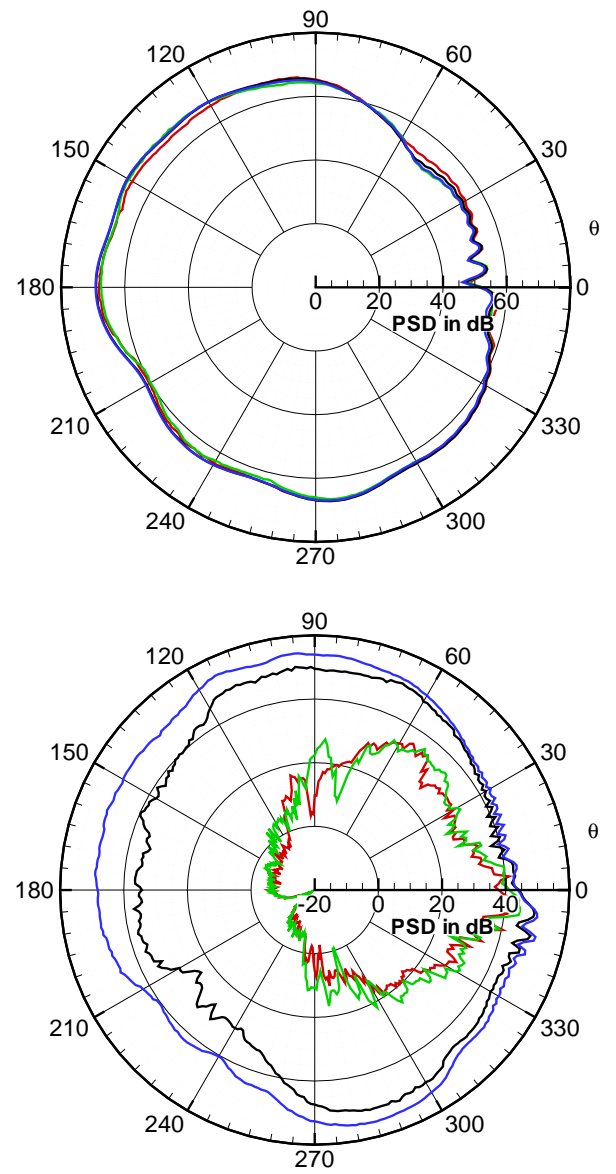

d)

Figure 10. Sound pressure level directivities computed for FREQUENZ profile along $r=1.5$ on different grids; a) overall sound pressure levels, b) PSD levels at $\mathrm{f}=10 \mathrm{kHz}$, c) PSD levels at $\mathrm{f}=15 \mathrm{kHz}$, d) PSD levels at $\mathrm{f}=20 \mathrm{kHz}$; please refer to section III.A.10 for an explanation of the absolute levels.

the very coarse grid evidences slightly increased amplitudes in the downstream region, e.g. at $\theta=45^{\circ}$, though. Obviously, but not unexpectedly, this grid does not resolve the refraction of sound waves in the main element's boundary layer as precisely as the other grids. But, nevertheless, its solution at $10 \mathrm{kHz}$ is still considered as quite useful. At higher frequencies, the deviations are largest upstream, where the sound waves are compressed by the mean flow field, recall figure 8 . If the criterion for the usability of a grid is taken to be a maximal level drop-off of, say, $3 \mathrm{~dB}$, the cut-off frequencies of both the coarse grids are approximately $10 \mathrm{kHz}$, while the medium-fine grid may be used up to $15 \mathrm{kHz}$. This corresponds to a required ratio of triangle edge length to resolvable wave length of about $L / \lambda \leq 1 / 2$. A ratio of $L / \lambda \leq 1 / 3$ as proposed earlier ${ }^{3}$ is obviously not necessary in practise, only if extremely accurate results are required. According to the $L / \lambda \leq 1 / 2$ rule, the fine grid should be applicable up to $20 \mathrm{kHz}$. Its directivity is indeed still quite smooth at this frequency compared to the directivities from the other grids.

\section{III.A.7. Sharpened Slat Trailing Edges}

To save computational time, sharpened slat trailing edges are usually employed in the DG computations. Originally, the FREQUENZ geometry has blunt slat edges, though: the actual (dimensionless) thickness of the upper and lower edge is $d \approx 0.0008$ and $d \approx 0.0012$, respectively. Sharpened edges are only used in the DG computations, the underlying RANS solution is based on the original geometry.

The applicability of sharpened slat trailing edges is proven through a variant of the medium-fine DG grid from table 3 with the original blunt slat edges. Figure 11 depicts the two grids in the region of the upper slat trailing edge. $E=42.881$ elements were required in the case of blunt edges, while only $E=42.561$ were 

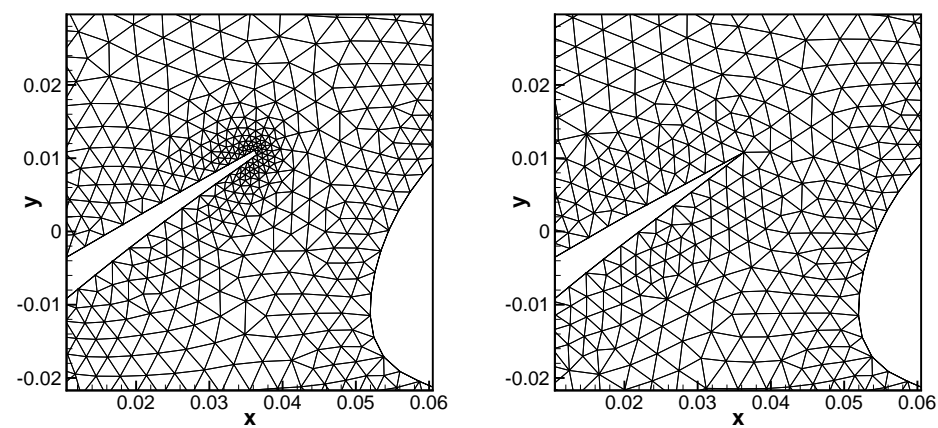

Figure 11. Comparison of medium-fine DG grids in vicinity of upper slat trailing edge of FREQUENZ geometry; left: blunt slat edges; right: sharp slat edges.

required in the case of sharp edges. Much more important, the tiny thickness of the blunt edges determined the minimum triangle edge length in the respective grid and thus also the time step size $d t .^{3}$ The maximum possible time step sizes according to the CFL condition times a safety factor of 0.8 were $d t=10.83 \cdot 10^{-5}$ for sharp edges, but only $d t=4.38 \cdot 10^{-5}$ for blunt edges, i.e., the computation with sharp edges was almost three times faster.

Figure 12 compares the sound pressure spectra obtained with blunt and sharpened slat trailing edges. To enable a better comparison, a time step of $d t=3.61 \cdot 10^{-5}=\frac{1}{3} 10.83 \cdot 10^{-5}$ instead of $d t=4.38 \cdot 10^{-5}$ was employed in the case of blunt edges. As also observed in Ref. 20, the solution indeed hardly changes through the sharpening.
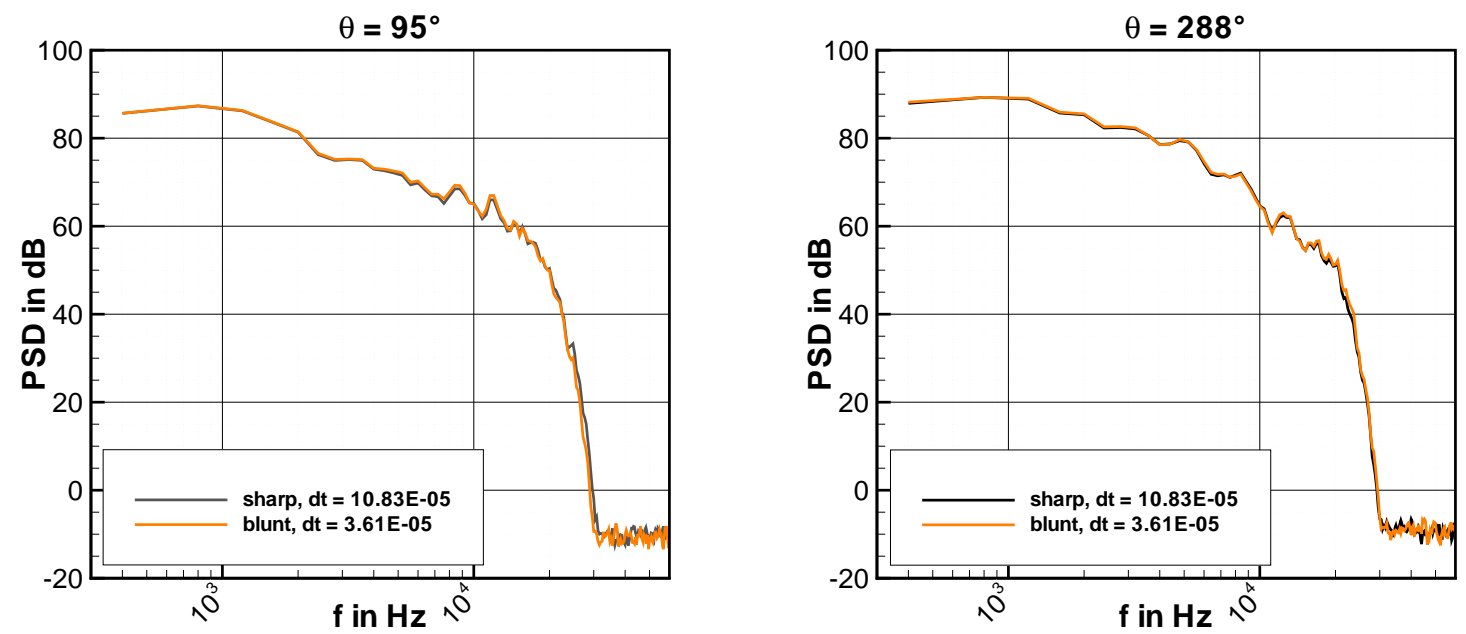

Figure 12. Comparison of sound pressure spectra computed for FREQUENZ geometry with blunt and sharpened slat trailing edges; left: receiving point at $(r, \theta)=\left(1.5,95^{\circ}\right)$; right: receiving point at $(r, \theta)=\left(1.5,288^{\circ}\right)$; please refer to section III.A.10 for an explanation of the absolute levels.

\section{III.A.8. FRPM Grid Resolution Studies}

Figure 13 shows sound pressure spectra obtained with the four different FRPM grids from table 2 using the medium-fine DG grid and a filter width of $l_{s}=0.003$. Simulations were performed both for $\hat{A}=\hat{A}\left(\boldsymbol{x}^{\prime}\right)$ as well as for $\hat{A}=\hat{A}(\boldsymbol{x})$. In both cases, the spectra from the first three FRPM grids are very similar, i.e., the coarse FRPM grid already delivers a rather grid-independent solution. This is remarkable, since these are Cartesian background grids, which do not specifically resolve turbulent shear layers. The spectra obtained on the very coarse FRPM grid notably deviate from the others, though. In particular, increased levels are 

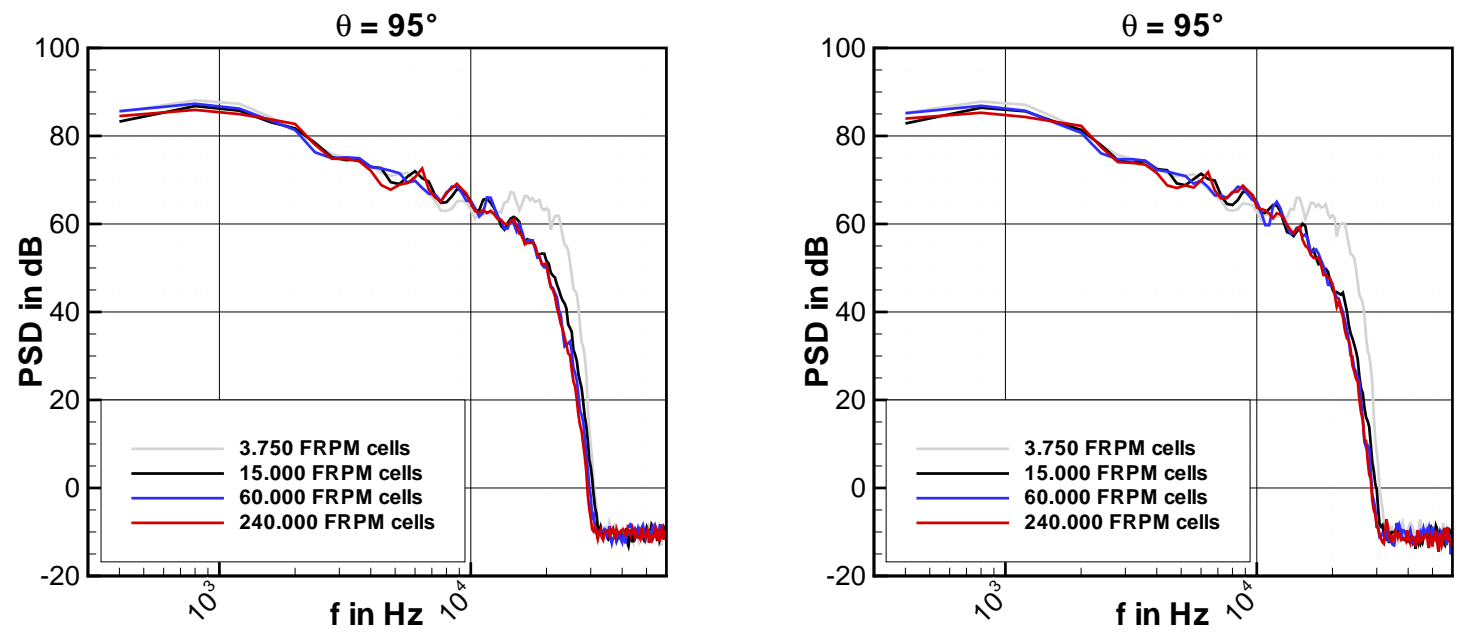

Figure 13. Sound pressure spectra at $(r, \theta)=\left(1.5,95^{\circ}\right)$ from FREQUENZ geometry using different FRPM grids and different local dependencies of the filter amplitude; left: $\hat{A}=\hat{A}\left(\boldsymbol{x}^{\prime}\right)$; right: $\hat{A}=\hat{A}(\boldsymbol{x})$; please refer to section III.A.10 for an explanation of the absolute levels.

observed at higher frequencies. This behaviour can not be explained at the moment and is unexpected, since high frequency amplitudes usually decrease, when a grid is too coarse, recall figure 9 . The differences between $\hat{A}=\hat{A}\left(\boldsymbol{x}^{\prime}\right)$ and $\hat{A}=\hat{A}(\boldsymbol{x})$ are very small, see right below.

\section{III.A.9. FRPM Parameter Studies}

Figure 14 investigates the influence of FRPM parameters on the acoustic result using the medium-fine FRPM grid in combination with the medium-fine DG grid. The left picture compares the influence of a local dependency $\hat{A}=\hat{A}\left(\boldsymbol{x}^{\prime}\right)$ or $\hat{A}=\hat{A}(\boldsymbol{x})$ of the filter amplitude $\hat{A}$. Obviously, there are hardly any differences up to $10 \mathrm{kHz}$. Beyond this frequency, increasingly higher levels are obtained with the formulation $\hat{A}=\hat{A}\left(\boldsymbol{x}^{\prime}\right)$. This behaviour can be explained by the fact, that the amplitude of the generated vorticity fluctuations $\omega_{3}^{\prime}$ is zero along walls in the case of $\hat{A}=\hat{A}(\boldsymbol{x})$, but not exactly zero in the case $\hat{A}=\hat{A}\left(\boldsymbol{x}^{\prime}\right)$. However, according to Howe's incompressible upwash velocity concept, ${ }^{9,17}$ the frequency of the sound generated at a trailing edge is inversely proportional to the distance of the turbulent eddies from the surface.

The right picture of figure 14 studies the influence of the filter width $l_{s}$. Evidently, this parameter controls the high-frequency content of the spectra: the lower $l_{s}$, the larger the levels at high frequencies. In general, the filter integral Eq. (4) does not account for objects, i.e., the vorticity fluctuations are actually non-zero within objects. Zero vorticity fluctuations within objects are e.g. realized by simply excluding respective FRPM grid points when the source is computed. In other words, vorticity fluctuations situated within objects are cut out. Now, the share of turbulent energy, which is thereby removed from eddies close to surfaces, is the bigger, the larger these eddies, i.e., the larger $l_{s}$. In combination with Howe's incompressible upwash velocity concept, this may explain the observations from figure 14 (right).

\section{III.A.10. Validation}

Computed spectra were compared to spectra measured by EADS Innovation Works in the framework of the FREQUENZ project in the AWB (Acoustic Windtunnel Braunschweig) of DLR at different microphone positions. Like in the simulations, the test object had a clean chord length of $L^{*}=0.4 \mathrm{~m}$. Its span was $L_{z}^{*}=0.8 \mathrm{~m}$, and the angle of attack in the measurements was set to $\alpha=23^{\circ}$ to obtain a similar pressure distribution as in the computation, i.e., in the RANS solution, where $\alpha=13^{\circ}$ was used. Measured spectra are juxtaposed to those from the computation with $l_{s}=0.0015$, figure 14 (right), which is, as mentioned above, based on the medium-fine FRPM grid consisting of 60.000 cells and a local dependency $\hat{A}=\hat{A}\left(\boldsymbol{x}^{\prime}\right)$ of the filter amplitude. Furthermore, the medium-fine DG grid, which consists of 42.561 elements and has a resolution limit of about $15 \mathrm{kHz}$, was used. 

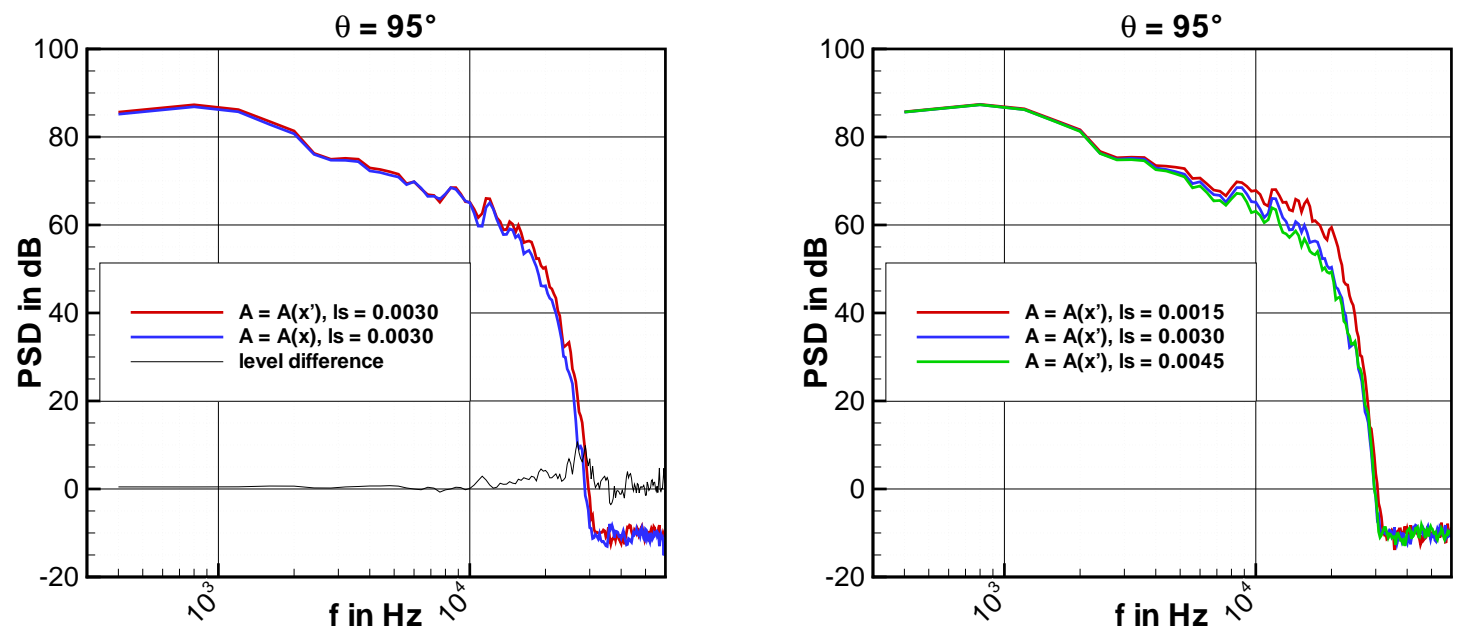

Figure 14. Sound pressure spectra at $(r, \theta)=\left(1.5,95^{\circ}\right)$ from FREQUENZ geometry using medium-fine FRPM grid and medium-fine DG-grid; left: $\hat{A}=\hat{A}\left(\boldsymbol{x}^{\prime}\right)$ vs. $\hat{A}=\hat{A}(\boldsymbol{x})$; right: $\hat{A}=\hat{A}\left(\boldsymbol{x}^{\prime}\right), l_{s}=0.0015,0.0030$, and $0.0045 ;$ please refer to section III.A.10 for an explanation of the absolute levels.

Spectra at $(r, \theta)=\left(1.5,95^{\circ}\right)$ and $(r, \theta)=\left(1.5,288^{\circ}\right)$ from the $2 \mathrm{D}$ computation were compared to spectra measured at $(r, \theta)=\left(2.14,95^{\circ}\right)$ and $(r, \theta)=\left(3.43,288^{\circ}\right)$, i.e., some corrections had to be applied to the computed spectra.

First, the distances of the receiving points were smaller than in the measurements. However, the shape of the computed spectra was found to be practically independent of distance $r$ for $r>1$. Thus, merely frequencyindependent level offsets $\Delta L_{p}$ had to be applied to the computed spectra at $r=1.5$ to obtain spectra at distances $r=2.14$ and $r=3.43$. These offsets were calculated in terms of the asymptotic $2 \mathrm{D}$ farfield decay law of the sound pressure, $p^{\prime} \propto r^{-0.5}$. The resulting offsets $\Delta L_{p}=10 \log \left(\frac{r_{\text {comp }}}{r_{\text {meas }}}\right) \mathrm{dB}$ were $\Delta L_{p}=-1.55 \mathrm{~dB}$ at $\theta=95^{\circ}$ and $\Delta L_{p}=-3.59 \mathrm{~dB}$ at $\theta=288^{\circ}$.

The second correction concerns the two-dimensionality of the computations. According to Ref. 11, a correction given by

$$
L_{p}(x, y)_{3 \mathrm{D}}=L_{p}(x, y)_{2 \mathrm{D}}+10 \log \left(\frac{C}{2 \pi} \frac{L_{z}}{r} M a\right)
$$

should be applied to the $2 \mathrm{D}$ spectra, where the constant $C$ relates the spanwise coherence length scale of the trailing edge source to that ot the surface pressure in the vicinity of the trailing edge, ${ }^{11} C \approx 2$. Thus, just a simple offset has to be applied to the spectral levels from the $2 \mathrm{D}$ computation. The offsets for $L_{z}=L_{z}^{*} / L^{*}=2.0$ and $M a=0.166$ were $\Delta L_{p}=-13.07 \mathrm{~dB}, \Delta L_{p}=-15.11 \mathrm{~dB}$ for $r=2.14, r=3.43$, respectively. Eq. (24) actually only holds in the high-frequency limit and only for broadband spectra without tonal components. Its derivation is based on the sound generation at the trailing edge of a semi-infinite thin flat plate, and it assumes statistically homogeneous flow in spanwise direction.

Third, another $16 \mathrm{~dB}$ were generally subtracted from computed levels to calibrate the FRPM-DG method, so that the final level offsets applied to the computed spectra were $\Delta L_{p}=-30.62 \mathrm{~dB}$ at $\theta=95^{\circ}$ and $\Delta L_{p}=-34.70 \mathrm{~dB}$ at $\theta=288^{\circ}$.

Figure 15 shows the resulting comparison of computation and measurement. There is is very good agreement at $\theta=95^{\circ}$ and satisfying agreement at $\theta=288^{\circ}$. Potential reasons for discrepancies are:

- The assumptions underlying the $2 \mathrm{D}$ to $3 \mathrm{D}$ correction are not completely fulfilled. For example, the sound field is not purely broadband due to acoustic resonances in the slat cove.

- The spectra measured at $\theta=288^{\circ}$ obviously contain excessive unwanted peaks between $1 \mathrm{kHz}$ and 3 $\mathrm{kHz}$. Similar observations are reported in Ref. 6, where they are explained by laminar vortex shedding off the lower slat edge. They could be avoided by some tripping upstream of the lower slat edge. ${ }^{6}$

- The reliability of the RANS results is unclear. A respective validation like e.g. through hot-wire measurements would be helpful. 

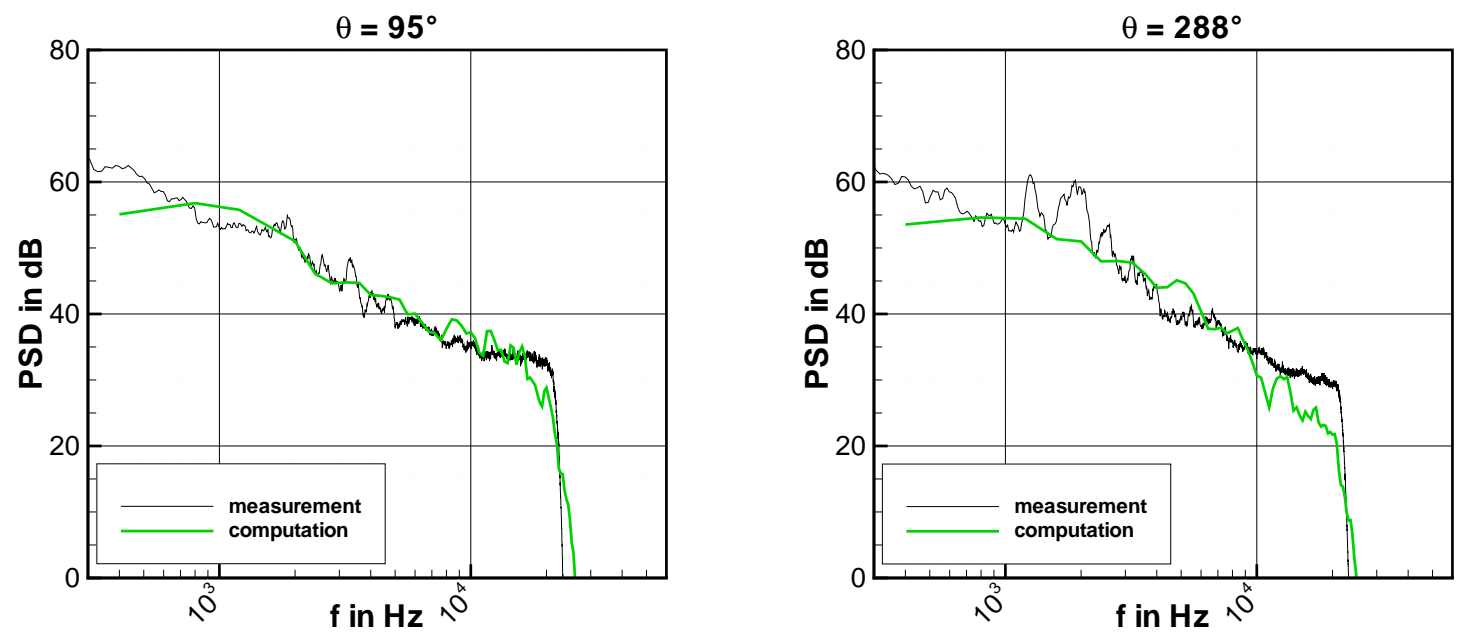

Figure 15. Comparison of spectra from computation and measurement at FREQUENZ geometry; 30.62 dB were subtracted from the computed spectra at $\theta=95^{\circ}$, and $34.70 \mathrm{~dB}$ at $\theta=288^{\circ}$.

- Only half the APE source vector was used as a first step, recall Eq. (3).

- There are some assumptions on the vorticity fluctuations generated by FRPM. For example, they are isotropic and have a spatially constant length scale $l_{s}$.

But, all in all, the agreement between computation and measurement is very encouraging.

\section{III.A.11. Computational Times}

Table 4 lists the computational times of the simulations from figure 9, where the four different DG grids were tested in combination with the medium-fine FRPM grid. A dimensionless time span of $t \approx 25$ was simulated, since this was found to be sufficient to calculate meaningful acoustic spectra with a resolution of $\Delta f \approx 400 \mathrm{~Hz}$. The Intel Fortran compiler was used, and the computations were run on a single AMD CPU of $2.8 \mathrm{GHz}$ clock rate. The employed PC has four such CPUs. The computational time of one and the same simulation may vary a little bit, depending on the workload from potential additional tasks.

Table 4. Computational times of FRPM-DG code for FREQUENZ simulations using different DG grids.

\begin{tabular}{l|l|l|l} 
fine grid & medium-fine grid & coarse grid & very coarse grid \\
\hline $85: 38 \mathrm{~h}$ & $42: 40 \mathrm{~h}$ & $16: 07 \mathrm{~h}$ & $8: 45 \mathrm{~h}$
\end{tabular}

Using the very coarse DG grid, the computation takes less than nine hours, and delivers useful results up to $10 \mathrm{kHz}$ and the correct overall sound pressure level! To assess the FRPM share $T_{\mathrm{FRPM}}$ of the overall computational time $T$, a computation without the DG time integration was performed on this very grid. This means, that only the new FRPM source plus its bilinear interpolation onto the respective DG nodes was computed at each time step. Then, the computational time was about 3:20h instead of 8:45h. This corresponds to a ratio of $T_{\mathrm{FRPM}} / T \approx 0.38$. $T_{\mathrm{FRPM}}$ is deemed rather independent of the employed DG grid, so that this ratio will probably be significantly lower on the finer DG grids.

Computational times may be reduced further by measures like:

- Parallelization of FRPM-DG code. Since the individual elements are very autonomous, the DG-method is well-suited for this. ${ }^{1,19}$

- Construction of more efficient DG grids. Maybe a coarser resolution is applicable in the source region or in the boundary layer along the main element. Regarding the far-field resolution, larger elements 
may be employed downstream of the source, where the wave length of the sound waves is larger than upstream.

- Use of local time steps instead of a common global time step for all elements. ${ }^{27}$

- Use of recursive Gaussian filters for FRPM. ${ }^{24}$

\section{III.B. TIMPAN High-Lift Airfoil Configurations}

\section{III.B.1. Foreword}

Next to the FREQUENZ geometry, high-lift airfoil configurations from the EU project TIMPAN were considered, too. They are three-element profiles consisting of an airfoil with a deployed slat and a deployed flap. The effect of different slat positions on the noise generated at the slat was investigated.

\section{III.B.2. Setup}

Figure 16 displays the coordinate systems. The airfoil configurations were situated in cold flow of free

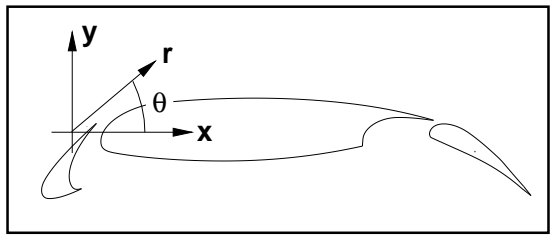

Figure 16. Coordinates used at TIMPAN geometries (image is not to scale).

stream Mach number $M a=0.175$ at an angle of attack of $\alpha=6^{\circ}$. The reference quantities used for non-dimensionalization according to Eqs. (22) were the stowed chord of $L^{*}=0.3 \mathrm{~m}$, as well as the density $\varrho_{\infty}=1.21 \frac{\mathrm{kg}}{\mathrm{m}^{3}}$ and the speed of sound $c_{\infty}=342 \frac{\mathrm{m}}{\mathrm{s}}$ at infinity. Accordingly, the Reynolds number based on $L^{*}$ was $R e \approx 1.2 \cdot 10^{6}$.

Computations were carried out for different values of gap and overlap between slat and main element, see figure 17 for an illustration of what is meant by "gap" and "overlap". Table 5 lists their values and introduces names for the respective configurations.
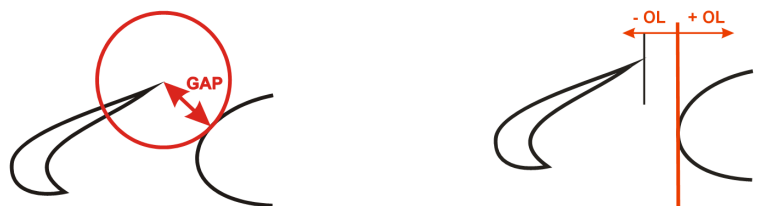

Figure 17. Illustration of gap and overlap; the smaller the overlap, the larger the distance between slat and main element.

Table 5. Dimensionless values of gap and overlap for investigated TIMPAN geometries.

\begin{tabular}{c|cc} 
name of geometry & gap & overlap \\
\hline reference & 0.02337 & -0.01085 \\
setting 1 & 0.02337 & -0.00500 \\
setting 2 & 0.01000 & -0.00500 \\
setting 3 & 0.01637 & -0.00500
\end{tabular}

The two-dimensional RANS mean flow fields were calculated by Dr. J. Held (department AS-CA) with the newly developed CFD-DG code PADGE (Parallel Adaptive Discontinuous Galerkin Environment) ${ }^{15,16}$ 
of DLR. The underlying grids all consisted of 25.760 quadrangles and had a farfield extension of about ten chord lengths. Like in case of the CAA-DG code, the solution was represented by polynomials of degree three in the individual elements. Thus, the order of accuracy was four in the CFD computations, too. The standard Wilcox $k-\omega$ turbulence model was employed. ${ }^{28}$

The Cartesian FRPM grid consisted of $321 \cdot 281=90.201$ points or 89.600 cells. Its extension was 0.160 in $x$-direction and 0.140 in $y$-direction, so that $\Delta x=\Delta y=0.0005$. It was anchored to the slat trailing edge. According to later experience from computations for the FREQUENZ geometry, this FRPM grid was probably finer than necessary, recall section III.A.8. Apart from a somewhat increased computational time, this did not cause any other disadvantages, though. Generously, $9.0 \cdot 10^{5}$ particles were used, and again the source term was smoothly faded in and out at the patch boundaries to avoid spurious noise. The constant filter length scale was set to $l_{s}=0.002$.

The circular DG grids all consisted of about 25.500 triangles and had a diameter of four. Their upper frequency limit was approximately $15 \mathrm{kHz}$. To save computational time, blunt edges were sharpened again.

\section{III.B.3. Results}

Figure 18 depicts an exemplary distribution of the Mach number and of the turbulent kinetic energy from PADGE, and figure 19 shows snapshots of the pressure perturbation field for settings 1 and 2 as computed
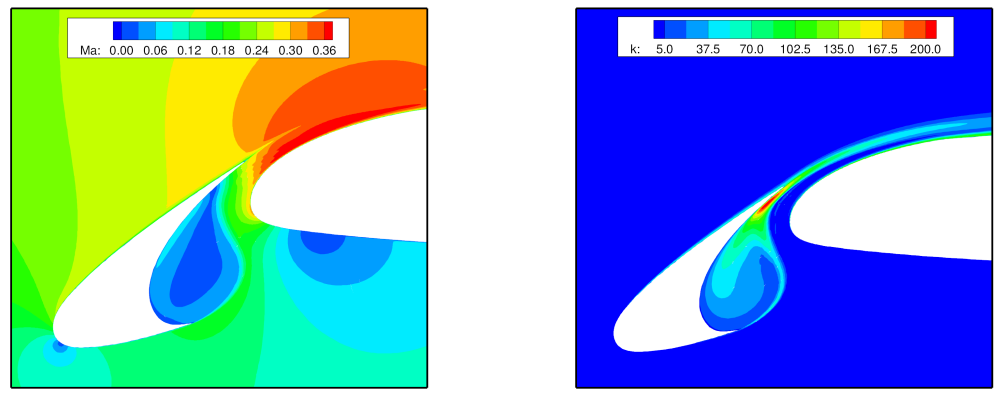

Figure 18. Exemplary distribution of Mach number (left) and of turbulent kinetic energy in $\mathrm{m}^{2} / \mathrm{s}^{2}$ (right) around slat of one of the TIMPAN geometries (images are not to scale).

on the DG grid. Obviously, the amplitude is lower in the case of setting 2, which has a smaller gap.
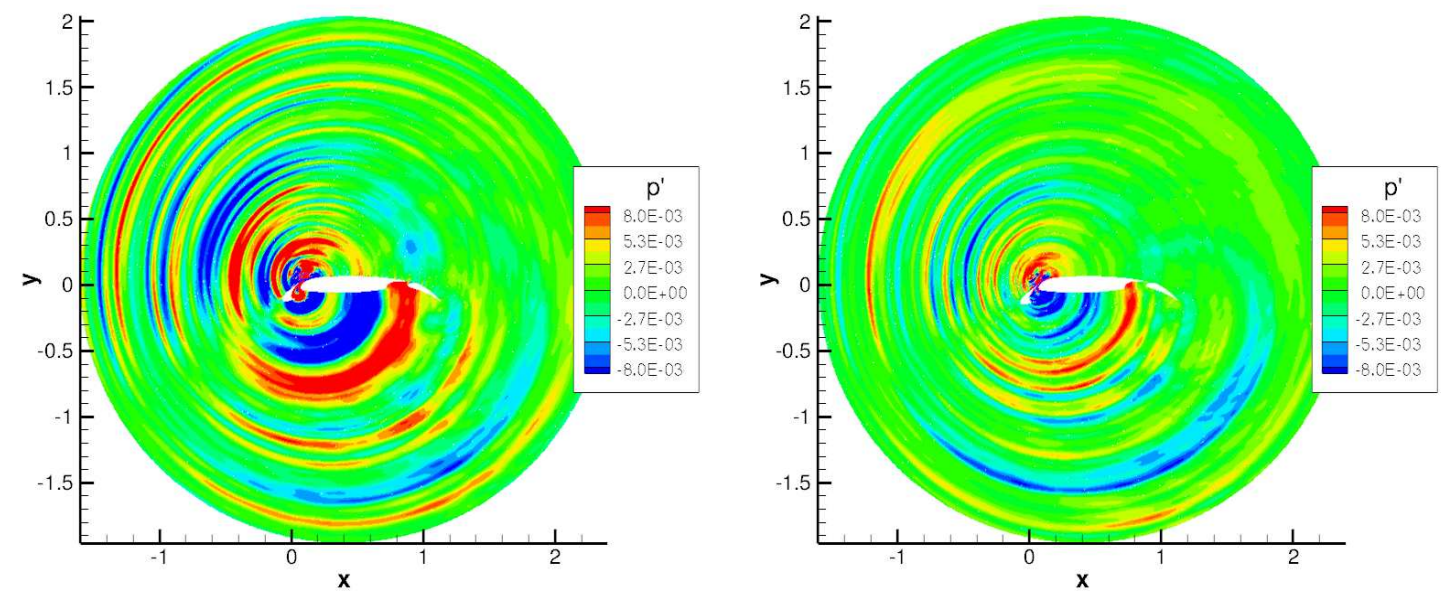

Figure 19. Instantaneous pressure perturbation field for setting 1 (left) and setting 2 (right) of the TIMPAN cases. 
Figure 20 compares sound pressure spectra from measurement and computation for settings 1-3. The measurements were conducted in the framework of the TIMPAN project in the Acoustic Windtunnel Braunschweig (AWB) of DLR. They indicate the noise to decrease as the gap becomes smaller. Thus, the rank order is: setting $1 \rightarrow$ setting $3 \rightarrow$ setting 2 . Principally, this rank order is also obtained in the computations. Deviations mainly occur around $6.5 \mathrm{kHz}$ and $10 \mathrm{kHz}$, where distinct peaks arise in the computations for setting 3 and especially for setting 2 . They may be explained by acoustic resonances in the slat cove: The smaller the gap, the lower their radiation losses, and the higher the amplitude of the associated spectral peaks. Resonances also occur in the measurements, but they seem to have a different behaviour regarding amplitude and frequency. This may be explained by the two dimensionality of the computations, which implies an unchanging solution in spanwise direction.
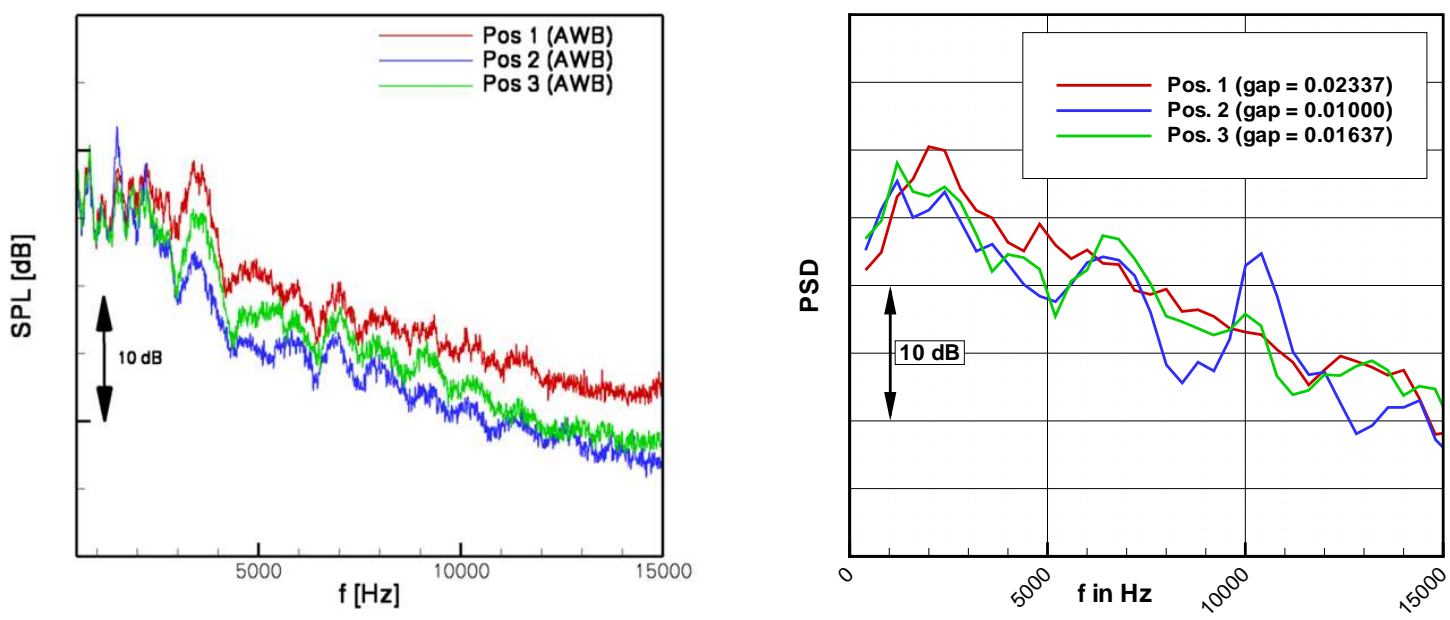

Figure 20. Comparison of measured (left) and computed (right) sound pressure spectra for TIMPAN cases 1-3 under an angle of $\theta=287^{\circ}$.

Each CAA simulation covered a dimensionless time span of $t \approx 30$ and took about 19 hours on one CPU of $2.8 \mathrm{GHz}$ clock rate. Since the employed PC has four such CPUs, three computations like e.g. for settings 1-3 were possible within one day, while one CPU was still available for other tasks.

\section{Conclusions and Outlook}

The agreement of computed and measured acoustic spectra is good. Part of the deviations may be attributed to the two dimensionality of the computations.

The employed FRPM grid does not have to be particularly fine, although it is only a background mesh, which is not body-fitted. Nearly identical solutions were obtained with different FRPM grids, except when a very coarse one was used, which only offered a handful of cells perpendicular to the flow direction to resolve the turbulence at the upper slat trailing edge. As soon as a decision has been made regarding the local dependency of the FRPM filter amplitude, the spatially constant filter width $l_{s}$ actually is the only parameter on the CAA side of the employed hybrid CFD/CAA approach. This parameter was found to control the high-frequency content of the acoustic spectra: the lower $l_{s}$, the larger the levels at high frequencies.

As far as the dependence of the acoustic result on the DG grid is concerned, a ratio of triangle edge length to acoustic wavelength of $L / \lambda \leq 1 / 2$ was found to be sufficient. A ratio of $L / \lambda \leq 1 / 3$ as proposed earlier $^{3}$ is actually only necessary for extremely accurate results. Blunt object edges may be sharpened when constructing the DG grid. The impact on computed result is very small, whereas the computational time may decrease significantly. Using sharpened edges and a good Fortran compiler, the proposed 2D FRPM-DG method can deliver meaningful predictions of high-lift systems' airframe noise within just one day. This is valid for a standard PC, and without parallelization of the code. No supercomputer or computer cluster is required. Thus, the 2D FRPM-DG method is well-suited for a cost-efficient design optimization, where 
numerous geometry variants have to be assessed within, say, a few weeks.

No stability problems have been encountered in any DG-APE computation so far. In contrast to Finite Difference schemes like the DRP scheme, ${ }^{5,25}$ the DG scheme does not need artificial selective damping ${ }^{26}$ to remain stable. Thus, the fine-tuning of the employed damping in the case of a new geometry does not apply and usually computations do not fail.

In the future, a 3D DGM on tetrahedra will be implemented. On the one hand, real complex geometries are inherently 3D and not 2D. On the other hand, the agreement of computed spectra with measurements may improve in 3D. Furthermore, the significance of the neglected part of the APE source vector should be investigated. The reliability of the RANS solutions underlying the FRPM-DG method may be another point of future interest.

\section{Acknowledgments}

This work was conducted in the framework of the DLR internal project AVANTGARDE. The two-element profile was taken from the German national project FREQUENZ, and the three-element profile from the EU project TIMPAN. The acoustic spectra of the two-element profile were measured by EADS-IW in the framework of the FREQUENZ project.

The authors would like to thank the TIMPAN consortium for kindly providing the related data, M. Pott-Pollenske for the measurements at the TIMPAN profile, Dr. J. Held for computing the mean flow field for this very profile, and C. K. Appel for computing the mean flow field for the FREQUENZ geometry.

\section{Appendix}

\section{Filter Amplitude}

The mean square $\hat{R}$ of the vorticity perturbations $\omega_{3}^{\prime}=\frac{\partial v^{\prime}}{\partial x}-\frac{\partial u^{\prime}}{\partial y}$ is

$$
\hat{R}=\overline{\omega_{3}^{\prime 2}}=\overline{\frac{\partial v^{\prime}}{\partial x} \frac{\partial v^{\prime}}{\partial x}}-2 \overline{\frac{\partial v^{\prime}}{\partial x} \frac{\partial u^{\prime}}{\partial y}}+\overline{\frac{\partial u^{\prime}}{\partial y} \frac{\partial u^{\prime}}{\partial y}},
$$

where the overbar denotes the temporal average as already mentioned in section II.C.1. According to Ref. 28, the dissipation per unit mass $\varepsilon$ is defined as

$$
\varepsilon=\nu \overline{\frac{\partial u_{i}^{\prime}}{\partial x_{k}} \frac{\partial u_{i}^{\prime}}{\partial x_{k}}}
$$

where $\nu$ is the kinematic molecular viscosity. In two dimensions this becomes

$$
\varepsilon=\nu\left(\overline{\frac{\partial u^{\prime}}{\partial x} \frac{\partial u^{\prime}}{\partial x}}+\overline{\frac{\partial u^{\prime}}{\partial y} \frac{\partial u^{\prime}}{\partial y}}+\overline{\frac{\partial v^{\prime}}{\partial x} \frac{\partial v^{\prime}}{\partial x}}+\overline{\frac{\partial v^{\prime}}{\partial y} \frac{\partial v^{\prime}}{\partial y}}\right)
$$

The terms $\frac{\overline{\partial v^{\prime}} \frac{\partial v^{\prime}}{\partial x}}{\partial x}$ and $\overline{\frac{\partial u^{\prime}}{\partial y} \frac{\partial u^{\prime}}{\partial y}}$ of $\hat{R}$ from Eq. (25) also appear in Eq. (27). Thus, $\hat{R}$ may as a first step roughly be estimated like

$$
\hat{R} \approx \frac{\varepsilon}{\nu}=\frac{0.09 \omega k}{\nu}
$$

where $\omega$ is the specific dissipation rate of turbulence, $k$ denotes the turbulent kinetic energy, and $\varepsilon=0.09 \omega k$ holds. $^{28}$ Note, that a very similar result is obtained through a more elaborate derivation. ${ }^{10}$ The only difference is an additional factor $4 / 15$, i.e.,

$$
\hat{R}=\frac{4}{15} \frac{0.09 \omega k}{\nu}
$$

The length scale of the vorticity fluctuations is related to the Taylor length scale $\lambda_{g},{ }^{21}$

$$
\lambda_{g}=\sqrt{10} \sqrt{\frac{\nu k}{\varepsilon}}
$$


which becomes quite small in boundary layers and in the slat cove. Thus, due to limited grid resolutions, a length scale $l_{s} \gg \lambda_{g, \min }, l_{s}=$ const., is used. To maintain the circulation of the turbulent eddies, an adjusted mean square value $\hat{R}^{*}$ of

$$
\hat{R}^{*}=\hat{R}\left(\frac{\lambda_{g}}{l_{s}}\right)^{2}
$$

has to be employed then. Substituting $\hat{R}$ and $\lambda_{g}$ according to Eq. (28) and Eq. (30), respectively, into Eq. (31) yields

$$
\hat{R}^{*} \approx \frac{k}{l_{s}^{2}}
$$

The filter amplitude $\hat{A}$ is finally obtained by requiring the mean square of the vorticity perturbations from FRPM, Eq. (4), to meet the prescribed value of $\hat{R}^{*}$. Assuming a constant filter amplitude $\hat{A}=$ const. or a dependency $\hat{A}=\hat{A}(\boldsymbol{x})$ for simplicity, the mean square of $\omega_{3}^{\prime}$ according to Eq. (4) can be written as

$$
\overline{\omega_{3}^{\prime} \cdot \omega_{3}^{\prime}}=\hat{A}^{2} \overline{\iint_{A_{s}} G_{0}\left(\boldsymbol{x}, \boldsymbol{x}^{\prime}\right) \mathcal{U}\left(\boldsymbol{x}^{\prime}, t\right) d \boldsymbol{x}^{\prime} \cdot \iint_{A_{s}} G_{0}\left(\boldsymbol{x}, \boldsymbol{x}^{\prime \prime}\right) \mathcal{U}\left(\boldsymbol{x}^{\prime \prime}, t\right) d \boldsymbol{x}^{\prime \prime}} .
$$

Considering that the first integral does not depend on $\boldsymbol{x}^{\prime \prime}$ gives

$$
\overline{\omega_{3}^{\prime 2}}=\hat{A}^{2} \overline{\iint_{A_{s}} G_{0}\left(\boldsymbol{x}, \boldsymbol{x}^{\prime \prime}\right) \mathcal{U}\left(\boldsymbol{x}^{\prime \prime}, t\right)\left[\iint_{A_{s}} G_{0}\left(\boldsymbol{x}, \boldsymbol{x}^{\prime}\right) \mathcal{U}\left(\boldsymbol{x}^{\prime}, t\right) d \boldsymbol{x}^{\prime}\right] d \boldsymbol{x}^{\prime \prime}} .
$$

Since $\mathcal{U}\left(\boldsymbol{x}^{\prime \prime}, t\right)$ is independent of $\boldsymbol{x}^{\prime}$ this becomes

$$
\overline{\omega_{3}^{\prime 2}}=\hat{A}^{2} \iint_{A_{s}} G_{0}\left(\boldsymbol{x}, \boldsymbol{x}^{\prime \prime}\right)\left[\iint_{A_{s}} G_{0}\left(\boldsymbol{x}, \boldsymbol{x}^{\prime}\right) \overline{\mathcal{U}\left(\boldsymbol{x}^{\prime}, t\right) \mathcal{U}\left(\boldsymbol{x}^{\prime \prime}, t\right)} d \boldsymbol{x}^{\prime}\right] d \boldsymbol{x}^{\prime \prime} .
$$

Substituting property Eq. (6) of the white noise field, splitting the two-dimensional filter kernels into a product of two one-dimensional kernels according to Eq. (9), assuming an infinite patch domain and making use of the sifting property obeyed by the Delta function leads to

$$
\overline{\omega_{3}^{\prime 2}}=\hat{A}^{2} \int_{-\infty}^{\infty} G_{1}^{x}\left(x^{\prime \prime}-x\right)^{2} d x^{\prime \prime} \cdot \int_{-\infty}^{\infty} G_{1}^{y}\left(y^{\prime \prime}-y\right)^{2} d y^{\prime \prime} .
$$

Considering the property

$$
\int_{-\infty}^{\infty} \frac{1}{\sqrt{2 \pi} \sigma} \exp \left[-\frac{z^{2}}{2 \sigma^{2}}\right] d z=1
$$

of the normal distribution, each of the integrals from Eq. (36) gives nothing but the length scale $l_{s}$, so that

$$
\overline{\omega_{3}^{\prime 2}}=\hat{A}^{2} l_{s}^{2} .
$$

Requiring this mean square value of the FRPM vorticity perturbations to meet the prescribed value of $\hat{R}^{*}=k / l_{s}^{2}$ according to Eq. (32) then yields

$$
\hat{A}=\frac{\sqrt{k}}{l_{s}^{2}}
$$

as stated in Eq. (10) above.

\section{Efficient Filtering}

The filtering can be performed very efficiently on the background mesh, since the two-dimensional Gaussian filter kernel can be split into a product of two one-dimensional kernels, recall Eq. (9). Using a somewhat streamlined notation, Eq. (20) thus becomes:

$$
\omega_{3}^{\prime}(i, j)=\sum_{l} \sum_{k} \hat{A}(k, l) G_{1}^{x}(i, k) G_{1}^{y}(j, l) \mathcal{W}(k, l) .
$$


Since $G_{1}^{y}$ does not depend on summation index $k$, this can be written as

$$
\omega_{3}^{\prime}(i, j)=\sum_{l} G_{1}^{y}(j, l) \underbrace{\sum_{k} \hat{A}(k, l) G_{1}^{x}(i, k) \mathcal{W}(k, l)}_{f(i, l)} .
$$

and the efficient filtering procedure reads:

1. Calculate the summations $f(i, l)=\sum_{k} \hat{A}(k, l) G_{1}^{x}(i, k) \mathcal{W}(k, l)$ for all grid points $(i, l)$ or $(i, j)$.

2. Calculate the summations $\omega_{3}^{\prime}(i, j)=\sum_{l} G_{1}^{y}(j, l) f(i, l)$ for all grid points $(i, j)$.

Since they are independent of index $j$, the very same values of $f(i, l)$ can be employed to calculate $\omega_{3}^{\prime}(i, j)$ at neighboring points $j$, i.e., the summations $f(i, l)$ are calculated only once. This kind of reuse provides the increased filter efficiency. A similar efficient filtering algorithm is also applicable in the case $\hat{A}=\hat{A}(\boldsymbol{x})$. A further speed-up could be obtained by realizing the 1D Gaussian filters through recursive filters. ${ }^{24}$

\section{References}

${ }^{1}$ H. L. Atkins and D. P. Lockard. A High-Order Method using Unstructured Grids for the Aeroacoustic Analysis of Realistic Aircraft Configurations. AIAA paper 99-1945, American Institute for Aeronautics and Astronautics, 1999.

${ }^{2}$ H. L. Atkins and C.-W. Shu. Quadrature-Free Implementation of Discontinuous Galerkin Method for Hyperbolic Equations. AIAA Journal, 36(5):775-782, 1998

${ }^{3}$ M. Bauer, J. Dierke, and R. Ewert. Application of a Discontinuous Galerkin Method to Predict Airframe Noise. AIAA paper 2009-3175, 2009

${ }^{4}$ M. Bauer, A. Zeibig, and P. Költzsch. Application of the SNGR-Model to Compute Trailing Edge Noise. http://www.ias.et.tu-dresden.de/akustik/Publikationen/Stroemungsakustik/Publikationen_STAK.htm. Research Report, Institute of Acoustics and Speech Communication, Dresden University of Technology, Germany, 2006.

${ }^{5}$ J. W. Delfs, M. Bauer, R. Ewert, H. A. Grogger, M. Lummer, and T. G. W. Lauke. Numerical Simulation of Aerodynamic Noise with DLR's Aeroacoustic Code PIANO (Version 5.2). Manual, Deutsches Zentrum für Luft- und Raumfahrt e.V., Institute of Aerodynamics and Flow Technology, 2008.

${ }^{6}$ W. Dobrzynski, K. Nagakura, B. Gehlhar, and A. Buschbaum. Airframe Noise Studies on Wings with Deployed High-Lift Devices. AIAA paper 1998-2337, American Institute for Aeronautics and Astronautics, 1998.

${ }^{7}$ K. Ehrenfried, C. Meyer, and A. Dillmann. Simulation of Sound Propagation in Boundary Layers based on Möhring's Acoustic Analogy. AIAA paper 2003-3272, American Institute for Aeronautics and Astronautics, 2003.

${ }^{8}$ R. Ewert. RPM - the Fast Random Particle-Mesh Method to realize unsteady turbulent Sound Sources and Velocity Fields for CAA Applications. AIAA paper 2007-3506, American Institute for Aeronautics and Astronautics, 2007.

${ }^{9}$ R. Ewert. Broadband Slat Noise Prediction based on CAA and Stochastic Sound Sources from a Fast Random ParticleMesh (RPM) Method. Computers and Fluids, 37(4):369-387, 2008.

${ }^{10}$ R. Ewert. Generation of Vorticity Fluctuations from RANS Results. Rough Sketch (unpublished), 2010.

${ }^{11}$ R. Ewert, C. Appel, J. Dierke, and M. Herr. RANS/CAA Based Prediction of NACA 0012 Broadband Trailing Edge Noise and Experimental Validation. AIAA paper 2009-3269, American Institute for Aeronautics and Astronautics, 2009.

${ }^{12}$ R. Ewert and R. Emunds. CAA Slat Noise Studies Applying Stochastic Sound Sources Based on Solenoidal Digital Filters. AIAA paper 2005-2862, American Institute for Aeronautics and Astronautics, 2005.

${ }^{13}$ R. Ewert, M. Meinke, and W. Schröder. Computation of Trailing Edge Noise via LES and Acoustic Perturbation Equations. AIAA paper 2002-2467, American Institute for Aeronautics and Astronautics, 2002.

${ }^{14}$ R. Ewert and W. Schröder. Acoustic Perturbation Equations based on Flow Decomposition via Source Filtering. Journal of Computational Physics, 188(2):365-398, 2003.

${ }^{15}$ R. Hartmann, J. Held, and T. Leicht. Adjoint-Based Error Estimation and Adaptive Mesh Refinement for the RANS and $k-\omega$ Turbulence Model Equations. J. Comput. Phys., 2010. Submitted.

${ }^{16}$ R. Hartmann, J. Held, T. Leicht, and F. Prill. Discontinuous Galerkin Methods for Computational Aerodynamics - 3D Adaptive Flow Simulation with the DLR PADGE Code. Aerospace Science and Technology, 2010. To appear.

${ }^{17}$ M. S. Howe. Trailing Edge Noise at Low Mach Numbers. Journal of Sound and Vibration, 225(2):211-238, 1999.

${ }^{18}$ D. König, S. R. Koh, W. Schröder, and M. Meinke. Slat Noise Source Identificaion. AIAA paper 2009-3100, 2009.

${ }^{19}$ D. P. Lockard and H. L. Atkins. Efficient Implementations of the Quadrature-Free Discontinuous Galerkin Method. AIAA paper 99-3309, American Institute for Aeronautics and Astronautics, 1999.

${ }^{20}$ D. P. Lockard and M. M. Choudhari. Noise Radiation from a Leading-Edge Slat. AIAA paper 2009-3101, 2009.

${ }^{21}$ S. Pope. Turbulent Flows. Cambridge University Press, 2000.

${ }^{22}$ P. P. Rao and P. J. Morris. Application of a Generalized Quadrature Free Discontinuous Galerkin Method in Aeroacoustics. AIAA paper 2003-3120, American Institute for Aeronautics and Astronautics, 2003.

${ }^{23}$ P. Rasetarinera, D. A. Kopriva, and M. Y. Hussaini. Discontinuous Spectral Element Solution of Acoustic Radiation from Thin Airfoils. AIAA Journal, 39(11):2070-2075, 2001.

${ }^{24}$ M. Siefert and R. Ewert. Sweeping Sound Generation in Jets Realized with a Random Particle-Mesh Method. AIAA paper 2009-3369, 2009. 
${ }^{25}$ C. K. W. Tam and J. C. Webb. Dispersion-Relation-Preserving Finite Difference Schemes for Computational Acoustics. Journal of Computational Physics, 107:262-281, 1993.

${ }^{26}$ C. K. W. Tam, J. C. Webb, and Z. Dong. A Study of the Short Wave Components in Computational Acoustics. Journal of Computational Acoustics, 1:1-30, 1993.

${ }^{27}$ J. Utzmann, T. Schwartzkopff, M. Dumbser, and C.-D. Munz. Heterogeneous Domain Decomposition for Computational Aeroacoustics. AIAA Journal, 44(10):2231-2250, 2006.

${ }^{28}$ D. C. Wilcox. Turbulence Modeling for CFD. DCW Industries, Inc., 1993. 